\title{
Spreading characteristics of an insoluble surfactant film on a thin liquid layer: comparison between theory and experiment
}

\author{
By ANNE D. DUSSAUD ${ }^{1} \dagger$, OMAR K. MATAR \\ AND SANDRA M. TROIA N \\ ${ }^{1}$ Unilever Research and Development, 40 Merritt Blvd, Trumbull, CT 06611, USA \\ anne.dussaud@unilever.com \\ ${ }^{2}$ Department of Chemical Engineering and Chemical Technology, Imperial College London, \\ London SW7 2AZ, UK \\ ${ }^{3}$ Department of Chemical Engineering, Princeton University, Princeton, NJ 08544, USA
}

(Received 14 December 2004 and in revised form 15 May 2005)

We describe measurements of the surface slope and reconstruction of the interface shape during the spreading of an oleic acid film on the surface of a thin aqueous glycerol mixture. This experimental system closely mimics the behaviour of an insoluble surfactant film driven to spread on a thin viscous layer under the action of a tangential (Marangoni) surface stress. Refracted image Moire topography is used to monitor the evolution of the surface slope over macroscopic distances, from which the time variant interface shape and advancing speed of the surfactant film are inferred. The interfacial profile exhibits a strong surface depression ahead of the surfactant source capped by an elevated rim at the surfactant leading edge. The surface slope and shape as well as the propagation characteristics of the advancing rim can be compared directly with theoretical predictions. The agreement is quite strong when the model allows for a small level of pre-existing surface contamination of the initial liquid layer. Comparison between theoretical and experimental profiles reveals the importance of the initial shear stress in determining the evolution in the film thickness and surfactant distribution. This initial stress appears to thin the underlying liquid support so drastically that the surfactant droplet behaves as a finite and not an infinite source, even though there is always an excess of surfactant present at the origin.

\section{Introduction}

The equilibrium characteristics of a laterally confined surface active film adsorbed at an air-liquid interface have been studied for well over a century beginning with Pockel's discovery in 1891 that fatty acid films on water attained a lower surface tension upon compression. Several years later, Rayleigh proposed that the molecules of the surface film floated on the water with little interaction until squeezed into contact at which point the surface tension was reduced (Adamson 1990). Since that time, experimental work has revealed the possibility of a variety of molecular orientations and equations of state describing the thermodynamic behaviour of surface films on a liquid support. The majority of work on the behaviour of surface active films at an air-liquid interface has focused on the equilibrium properties of a film under compression. By contrast, there have been relatively fewer studies of the non-equilibrium 
or dynamical behaviour describing the spreading of surfactant films. In practice, nonequilibrium conditions often prevail and many industrial and biological applications rely heavily on the rapid transport of surface active material along a liquid surface or moist substrate.

A theoretical model which can predict the behaviour of a spreading surfactant film would prove useful in many contexts. A successful model can lend insight into a physical system which is not so easily obtainable from experiment, such as the surface distribution of material, the liquid deformation that accompanies the spread of surfactant, or the way in which the surfactant delivery process affects the rate of mass transfer along the surface. The answer to these questions cannot be obtained from equilibrium measurements on compressed films. For example, although there has been much work devoted to elucidating the equations of state for long chained molecules under compression, these equations probably bear little relevance to the molecular configuration assumed during the spontaneous and rapid expansion of a surfactant monolayer. In what follows, we use the term 'surfactant' to describe any surface active material, including hydrocarbon films, which can sustain gradients in surface concentration during spreading and which lower the surface tension of the underlying liquid support.

Most of the experimental studies designed to probe the dynamics of spreading of a surfactant film have focused on deep-layer conditions (Fay 1969; Hoult 1972; Harper \& Dixon 1974; Huh, Inoue \& Mason 1975; DiPietro, Huh \& Cox 1978; Foda \& Cox 1980; Dagan 1984; Camp \& Berg 1987; Harper 1992; Jensen 1995). In this limit, the surfactant film spreads on a rather thick liquid support. The spreading surfactant exerts a tangential stress (Marangoni stress) which produces a viscous boundary layer in the underlying liquid support. The depth of the liquid support far exceeds the characteristic thickness of the boundary layer. For the most part, experiments have confirmed the theoretical predictions for the surface velocity. Recent work describing the surface deformation accompanying the surfactant transport at the air-liquid interface has not yet been tested experimentally (Jensen 1995). Even in the deep-layer limit, however, the theoretical model has considered only the spreading of a single-component insoluble non-volatile surfactant film. The equations have yet to be extended to include solubility, evaporation or multi-component surfactant mixtures, except for the work of Harper and Dixon (Harper \& Dixon 1974; Harper 1992) which allow for solubility.

Our interest lies in the other limit, the so-called lubrication, type flows, wherein the longitudinal extent of spreading far exceeds the thickness of the liquid support. These flows have also received attention because of their prevalence in coating or wetting processes. Ahmad \& Hansen (1972) performed some of the first experiments to confirm the speed of advance of a surfactant film on a thin liquid layer. The tangential stress condition applied to an air-liquid interface predicts that a surfactant monolayer spreading from a source at constant concentration (infinite reservoir) in rectilinear geometry should advance according to $L(t) \sim\left(2 h_{o} \Pi t / \mu\right)^{1 / 2}$, where $h_{o}$ denotes the undisturbed thickness of the liquid support, $\Pi$ denotes the maximum difference in surface tension between the clean and surfactant-coated film, $\mu$ is the viscosity of the liquid layer, and $t$ is time. This relation, which assumes that the tangential shear stress controls the spreading dynamics, is only valid for insoluble non-volatile surfactant films. Ahmad \& Hansen tested this relation by monitoring the spontaneous advance of an oleic acid film on the surface of a thin glycerol layer (whose thickness ranged from $0.32 \mathrm{~mm}$ to $1.21 \mathrm{~mm}$ ) contained in a long rectangular channel. Talc particles were first sprinkled on the undisturbed glycerol surface. A droplet of oleic acid was then 
gently placed on the glycerol layer from which a molecularly thin film was observed to spread rapidly and spontaneously. The oleic acid pushed against the talc particles compressing them into a visible line which marked the location of the advancing film as a function of time. The data agreed remarkably well with the expression for $L(t)$ given above. The authors noted in passing that, during the spreading, there developed 'a very noticeable height depression' in the vicinity of the oleic droplet beyond which the film thickness appeared nearly constant in height.

Gaver \& Grotberg (1992) performed similar experiments to determine the surface velocity throughout the extent of the spreading oleic film. They conducted their studies in a circular Petri dish containing a thin glycerol layer of two different thicknesses $(0.7 \mathrm{~mm}$ or $2.0 \mathrm{~mm})$. In order to deliver the surfactant as a true monolayer film, they first dissolved the oleic acid in hexane and spread this mixture inside a restraining ring which was placed within the glycerol layer. The hexane evaporated quickly leaving behind a monolayer of oleic acid. The spreading process was begun by lifting the ring manually. The authors indicated that this deposition procedure mimicked more closely their theoretical model describing the spreading of an insoluble surfactant monolayer. The surface velocity of the spreading film was inferred from the displacement of small dyed glycerol markers situated at various distances from the restraining ring. The effective Bond number, Bo $=\rho g h_{o}^{2} / \Pi$, in these experiments was not insignificant and contributed to the flow behaviour observed.

Gaver \& Grotberg (1990) compared their experimental results for the trajectory of the dye markers with their theoretical predictions for the spontaneous spreading of a monolayer under the action of Marangoni and hydrostatic forces. Comparison of the location of the convection front as a function of time yielded favourable agreement. Since Marangoni as well as hydrostatic effects contributed to the spreading rate, the exponent characterizing the location of the convection front was measured and predicted to be in the range 0.63 to 0.70 . The authors found, however, that the position of the surface convection front as predicted by the model, differed significantly from the position of the surfactant leading edge. This work provided more evidence that the model equations developed in the literature could successfully describe the spreading process. Other studies in the literature have also examined the dynamics of a spreading surfactant film with a view to measuring the exponent associated with the leading edge (Hussain, Fatima \& Ahmad 1975; Fraaije \& Cazabat 1989). Bull et al. (1999) and Bull \& Grotberg (2003) conducted experiments which involve the spreading of surfactant on thin pre-contaminated viscous films. In these studies, the deformation of the liquid film was measured by projecting a grid of light on the film surface and the surfactant leading edge was tracked by exploiting the fluorescence properties of the added surfactant; a restraining collar was also used in these studies to deliver the surfactant. A comparison of the theoretical predictions with experimental data yielded very good agreement. (Note also that experimental studies have also been conducted, which have focused on the fingering instability that accompanies the spreading of surfactant on thin films (Marmur \& Lelah 1981; Troian, Wu \& Safran 1989; Stoebe et al. 1997a, b; Cachile \& Cazabat 1999; Cachile et al. 1999, 2002; Fisher, Darhuber \& Troian 2001; Nikolov et al. 2002; Afsar-Siddiqui, Luckham \& Matar 2003a, b), a feature not considered in the present work.)

In this paper we present measurements of the surface deflection, the spreading coefficient, and the exponent describing the advance of an oleic acid film on the surface of a thin glycerol-water mixture. Instead of quantifying the flow with surface markers, we visualize the behaviour of the deformed surface using refracted-image Moiré topography (Kafri \& Glatt 1989). This sensitive technique, which maps the 
time variant surface slope, is ideally suited to surface deformations occurring over an extended region. The technique has been successfully used previously to measure the acoustical properties of adsorbed polymer monolayers at an air-water interface (Scott \& Stephens 1972), the wavelength and damping coefficient of propagating surface waves in the presence of surfactant (Scott 1972) and the surface profile of a falling liquid curtain (Kheshgi \& Scriven 1983). Note that our experimental set-up does not involve use of a restraining ring for surfactant delivery, which may be disruptive to the spreading dynamics at early times, particularly for the thin films investigated. We also do not pre-contaminate the thin liquid films prior to the spreading experiments. Furthermore, the surface deflection in our work is measured using the two-dimensional Moire fringe displacement and the interfringe of the undisturbed Moire pattern, which is limited only by the pixel size of the camera. This measurement technique, therefore, does not rely on the measurement of the projection angle of light (Bull \& Grotberg 2003), which is potentially difficult to measure accurately.

Following the presentation of our experimental results we make detailed comparison with the surface slope and spreading speed obtained from a theoretical model describing the spreading of a surfactant monolayer in the lubrication limit. The model successfully captures the spatio-temporal behaviour of the surface slope and the temporal dependence of the spreading surfactant film when a very small amount of surface contamination on the pre-existing liquid film was taken into account. The experimental technique described offers a convenient and sensitive tool for studying the time-variant waveform accompanying the spreading of surfactant on a thin liquid layer.

\section{Experimental procedure}

\subsection{Materials}

The liquid substrate consisted of an aqueous mixture of $60 \%$ wt glycerol with tabulated values (Jungermann \& Sonntag 1991) of the viscosity, density and index of refraction equal to $\mu^{*}=0.107$ poise, $\rho^{*}=1.15 \mathrm{~g} \mathrm{~cm}^{-3}$ and $n=1.41$ at $20^{\circ} \mathrm{C}$, respectively. The glycerol $(99.5+\%$, Aldrich) was used as received from the supplier. The water used for cleaning glassware and syringes as well as making the liquid mixtures was distilled de-ionized ultra-pure water with a resistivity of $(18 \mathrm{M} \Omega \mathrm{cm})$ supplied from a HYDRO picosystem unit. The surface tension and spreading pressure of the liquids used were measured with a platinum Wilhelmy plate suspended from a Denver Instrument electro-balance with a resolution of $0.5 \mathrm{mg}$. The surface tension of the ultra-pure water was measured to be $72.2 \pm 0.3 \mathrm{dyn} \mathrm{cm}^{-1}$ at $23^{\circ} \mathrm{C}$, in good agreement with tabulated values. The average surface tension of the aqueous glycerol mixture was measured to be $62.3 \pm 0.03 \mathrm{dyn} \mathrm{cm}^{-1}$ at $23^{\circ} \mathrm{C}$, somewhat lower than the value of $68.4 \mathrm{dyn} \mathrm{cm}^{-1}$ at $18^{\circ} \mathrm{C}$ reported by Lim \& Berg (1975). The difference of $5 \mathrm{~K}$ between the two measurements can only account for a difference of $0.7 \mathrm{mN} \mathrm{m}^{-1}$. The discrepancy indicates that the glycerol probably contained surface active impurities. The insoluble surfactant chosen for this study was oleic acid (octadecanoic acid, FW 282.47, $99+\%$, Aldrich), used as received from the supplier. Oleic acid monolayers are only sparingly soluble in an acidic aqueous glycerol subphase (Lim \& Berg 1975; Tomoaia-Cotisel et al. 1987). The relevant spreading pressure was measured according to the following procedure. The Wilhelmy plate was first lowered into a circular beaker $(9 \mathrm{~cm}$ in diameter and $5 \mathrm{~cm}$ in depth) containing the aqueous glycerol mixture and placed far from the centre and edges of the container. A droplet of oleic acid was then gently deposited at the centre of the liquid surface. The oleic acid droplet assumed the shape of a floating lens from which a microscopic film was observed to spread rapidly across the entire surface of the aqueous glycerol 
layer. After the readings had equilibrated, the surface tension registered a value of $40.2 \pm 0.01 \mathrm{dyn} \mathrm{cm}^{-1}$. From this information, the spreading pressure, $\Pi^{*}$, was estimated to be $62.3-40.2=22.1 \mathrm{dyn} \mathrm{cm}^{-1}$. The talc powder (Aldrich) which was used in some experiments to mark the front of the spreading oleic acid, was first purified by heating to $400^{\circ} \mathrm{C}$ overnight to remove organic contaminants.

\subsection{Deposition procedure}

The experiments were performed in a circular glass dish $19 \mathrm{~cm}$ in diameter and $0.2 \mathrm{~cm}$ in depth which was fitted with an optically flat bottom. All parts of the dish and syringe system used to deliver the oleic acid were cleaned according to published procedures (Dussaud \& Troian 1997). It was especially important in these studies to ensure a high degree of cleanliness and wettability of the glycerol mixture against the sides and bottom of the glass dish since any organic contaminant on the glass surface would quickly nucleate a dewetted region, ruining the uniformity of the initial liquid layer. This was especially problematic in working with liquid films in the range of $250 \mu \mathrm{m}$ or less. The thickness of the initial liquid layer was estimated by pouring a fixed volume of liquid into the glass cell and dividing by the measured surface area of the dish bottom. A $7 \mathrm{~cm}^{3}$ volume produced a liquid film of approximately $250 \mu \mathrm{m}$ in thickness. This estimate did not include the fact that the interior bottom edge of the dish, where the optically flat bottom had been fused to the sidewall, was slightly curved. In addition, the glycerol-water mixture was poured from a glass burette which retained a thin wetting film after being emptied in the glass dish. Finally cleanliness and wettability of the glass dish, while enhancing the spreading capability of the mixture throughout the dish bottom, also enhanced the wettability of the sidewalls and encouraged a thin wetting film to climb upwards. The error on the film thickness was estimated to be approximately $4 \%$.

Immediately after filling the glass dish with the glycerol mixture, a droplet of oleic acid with volume $0.25 \mu \mathrm{l}$ or $0.50 \mu \mathrm{l}$ was gently deposited on the surface from a micrometer-controlled assembly described previously (Dussaud \& Troian 1997). The droplet was delivered from a precision $100 \mu \mathrm{l}$ Hamilton digital syringe mounted with a fine Teflon needle (OD $0.81 \mathrm{~mm}$ ) to ensure complete expulsion. A curved glass Pasteur pipette (OD $1.6 \mathrm{~mm}$ ) was used to guide the length of the Teflon needle. Typical sideview pictures of a $0.25 \mu \mathrm{l}$ oleic droplet immediately before and after deposition on an aqueous glycerol layer of $250 \mu \mathrm{m}$ thickness are shown in figures $1(a)$ and $1(b)$. For the experiments in which talc was used to visualize the location of the surfactant front, the particles were sprinkled on the undisturbed liquid surface from a fine stainless steel sieve.

\subsection{Visualization technique}

A schematic diagram of the optical arrangement used is shown in figure 2 . The system rested on a vibration-free optical table to shield the liquid filled dish from disturbances which would cause capillary waves. The glass dish, which lay on a three-point adjustable level stage, was illuminated from below by a collimated beam of light from a $10 \mathrm{~mW} \mathrm{He}-\mathrm{Ne}$ laser. The light was passed through a spatial filter, a beam expander and a plano-convex lens $(f=60 \mathrm{~cm})$ to produce a uniformly lit area $9 \mathrm{~cm}$ in diameter. The beam then traversed two linear gratings, $G_{1}$ and $G_{2}$ (see figure 3), consisting of two identical square glass Ronchi rulings $\left(9 \times 9 \mathrm{~cm}^{2}\right)$ of pitch $250 \mu \mathrm{m}$ (Edmund Scientific). Moire fringes were obtained by projecting the shadow of the illuminating grating, $G_{1}$, located below the glass dish, onto the reference grating, $G_{2}$, positioned at an adjustable distance, $d$, above the liquid surface. The two gratings were mounted in rigid holders that could be rotated to produce a relative opening angle of $\theta$. One 
(a)

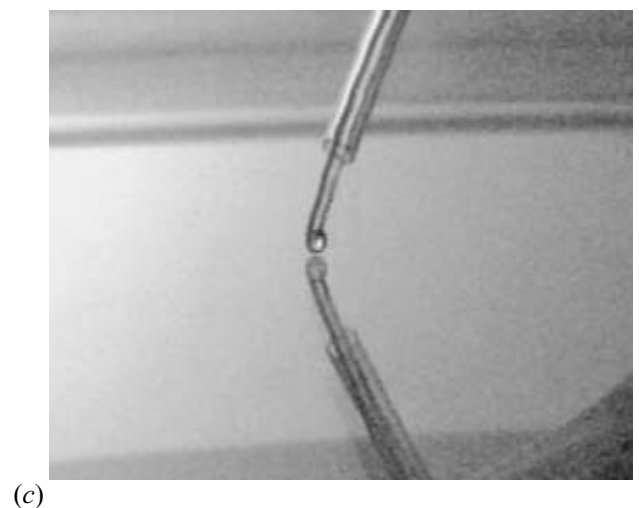

(c)

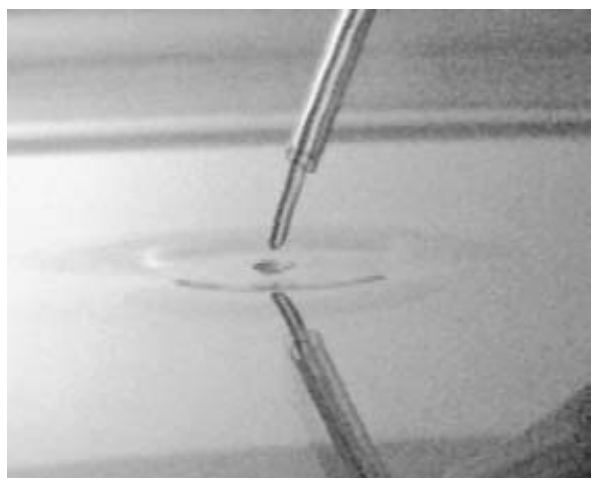

(b)
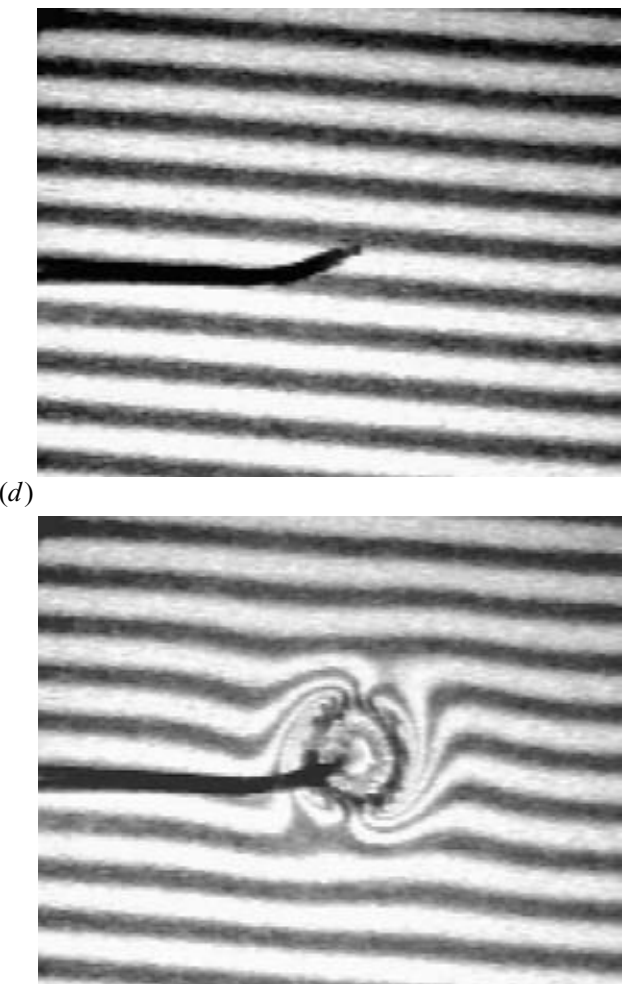

FIgURE 1. $(a, b)$ Side view and Moiré image of oleic acid droplet suspended above a thin aqueous glycerol layer respectively. $(c, d)$ Side view and Moire image of the surface deformation $0.3 \mathrm{~s}$ after deposition respectively. Teflon tubing of $\mathrm{OD}=0.81 \mathrm{~mm}$ is guided through a curved glass housing of $\mathrm{OD}=1.6 \mathrm{~mm}$. Undisturbed interfringe spacing $i=4.78 \mathrm{~mm}$.

camera (Dage MTI VE 1000 CCD) was focused on the combined fringe pattern displayed on a miniature projection screen. This screen consisted of a sheet of translucent, high quality rag vellum which had been placed in contact with the outer surface of the second grating, $G_{2}$. A second camera (Cohu 4910 CCD) was focused on the syringe tip which delivered the oleic acid droplet to the liquid surface. This second camera was used to ensure that the droplet delivery was instantaneous and complete and that the syringe tip did not accidentally puncture the liquid surface. The region near the syringe tip was illuminated by a soft diffuse light produced by shining a fibre optic lamp onto a square sheet of vellum. This additional light source had no discernible effect on the Moire pattern. There are many excellent references describing the geometric optics that govern the Moiré technique in the refracted image configuration (Theocaris \& Koutsabessis 1965; Scott 1972; Scott \& Stephens 1972; Kheshgi \& Scriven 1983; Kafri \& Glatt 1989). We therefore simply list the relevant equations used to reconstruct the time-variant surface profile in the Appendix and turn our attention to the experimental results.

\section{Experimental results}

\subsection{Measurement of surface deflection}

Figure 1(c) depicts the surface deformation of the liquid support $0.30 \mathrm{~s}$ after droplet deposition. The liquid layer is strongly thinned into a flat disk which is capped by 


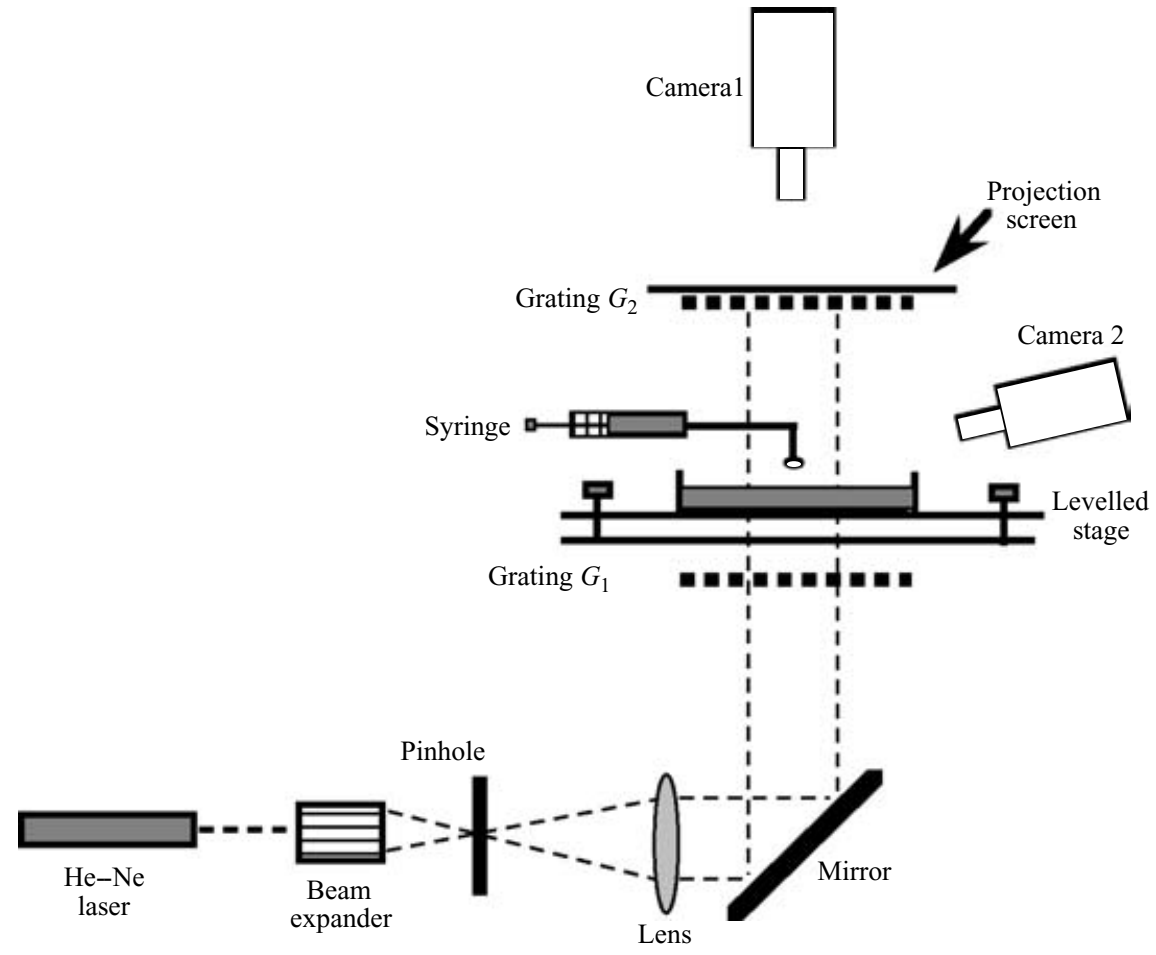

FIGURE 2. Schematic drawing of experimental apparatus (not to scale).

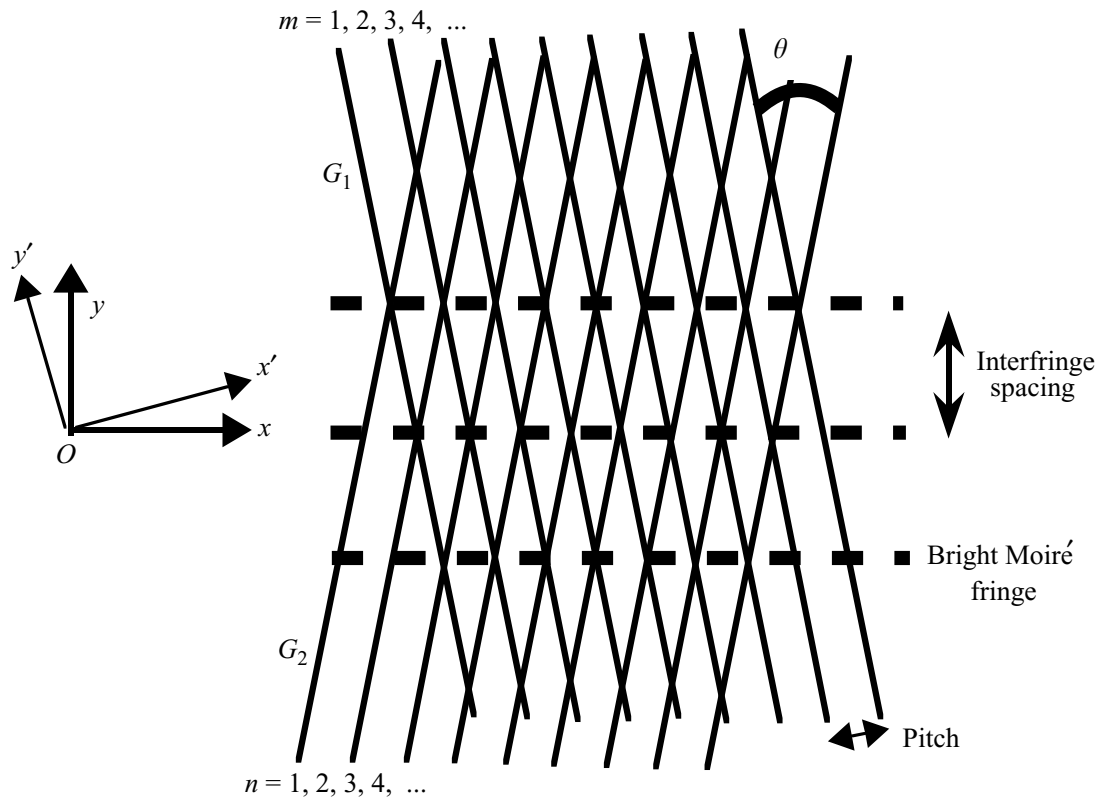

Figure 3. Formation of Moiré fringes by two line gratings, $G_{1}$ and $G_{2}$, of equal pitch, $p$, with opening angle $\theta$.

a thickened rim. At the origin, there remains an excess of oleic acid which assumes the shape of a hemispherical droplet. The distorted fringes in figure $1(d)$ demarcate the regions which undergo strong deformation, $\alpha=\partial h / \partial x^{\prime}$, where $x^{\prime}$ is the direction 
perpendicular to the grating lines (see figure 3). The central white spot corresponds to the residual oleic acid droplet. The black ring corresponds to a region of very high curvature located at the back of the advancing rim in figure $1(c)$. As the relation (9) (see Appendix A) which relates the surface deformation and the Moire fringe displacement is only valid for deformation in one direction, the only fringe line which was analysed to determine the surface slope of the deformed liquid layer is the line passing through the centre of the deposited droplet. Since the distortion near the black ring is quite large and the fringe displacement difficult to measure, the tracking was halted at the outermost edge of the black ring.

For centred depositions, the fringes on the left- and right-hand sides of the droplet were analysed for the surface reconstruction. A mouse-driven cursor was used to follow the deformation of a fringe from its undisturbed position by tracking the edge between the black and white areas. The cursor size was approximately one pixel in extent and did not obscure any features of the fringe pattern. Repeated measurements on the same line introduced an error of no more than three pixels in length, which translated into an error in the surface slope of 0.0028 (for $d=2 \mathrm{~cm}$ ) and 0.0012 (for $d=7 \mathrm{~cm}$ ). The position of an undisturbed fringe, which was required for calculating the displacement, $\delta y$, as well as the interfringe spacing, $i$, was determined from an undisturbed Moiré pattern (figure $1 b$ ) obtained prior to deposition by averaging over three independent frames. The data for the surface slope as a function of the distance from the drop centre was linearly interpolated to yield a smooth curve which was then integrated using a trapezoidal rule with a step size of $0.01 \mathrm{~cm}$ to produce the interface profile. The integration of the surface slope proceeded from right to left, commencing downstream of the spreading film where the Moire lines are perfectly parallel. The data obtained from the downstream portion of the advancing oleic acid front produced very small values of the surface slope. Depending on the choice of starting point in the integration of the surface slope, a small vertical shift in the film thickness could be obtained. We therefore repeated the integration of each run with different starting points to minimize this effect.

The sample curves in figure 4 reveal the typical form of the surface slope and the integrated interfacial profile for an oleic acid film spreading on a thin aqueous glycerol layer $0.3 \mathrm{~s}$ after deposition. The origin of the horizontal axis is coincident with the centre of the deposited droplet. The level 0 corresponds to the level of the undisturbed free surface. The two sets of data in figure 4(a) represent measurements of the surface slope from the left- (filled circles) and right-hand sides (open circles) of the same spreading droplet. The minimum in the surface slope, labelled $A$, corresponds to a location in the film thickness just ahead of the peak in the advancing rim. The maximum of the surface slope, corresponds to the outermost edge of the black ring demarcating the region of very high curvature which is connected to the very thin flat film formed around the droplet. As shown in figure 4(b), the liquid film suffers a strong depression ahead of the deposited droplet which rises sharply up to a peak and then decays over an extended region to meet the undisturbed film thickness. The region under the peak resembles an advancing rim. Gentle capillary oscillations sometimes appear ahead of the advancing rim, although these were not measurable because the Moire fringes they produce were not sharp enough. Since the fringe tracking was halted at the outer edge of the dark ring in figure $1(d)$, the exact degree of thinning ahead of the droplet cannot be accurately reported. The experimental quantities of interest used to compare with theoretical predictions are the maximum and minimum values in the slope, the propagation of the slope minimum, and the shape of the deformed film as a function of time. 

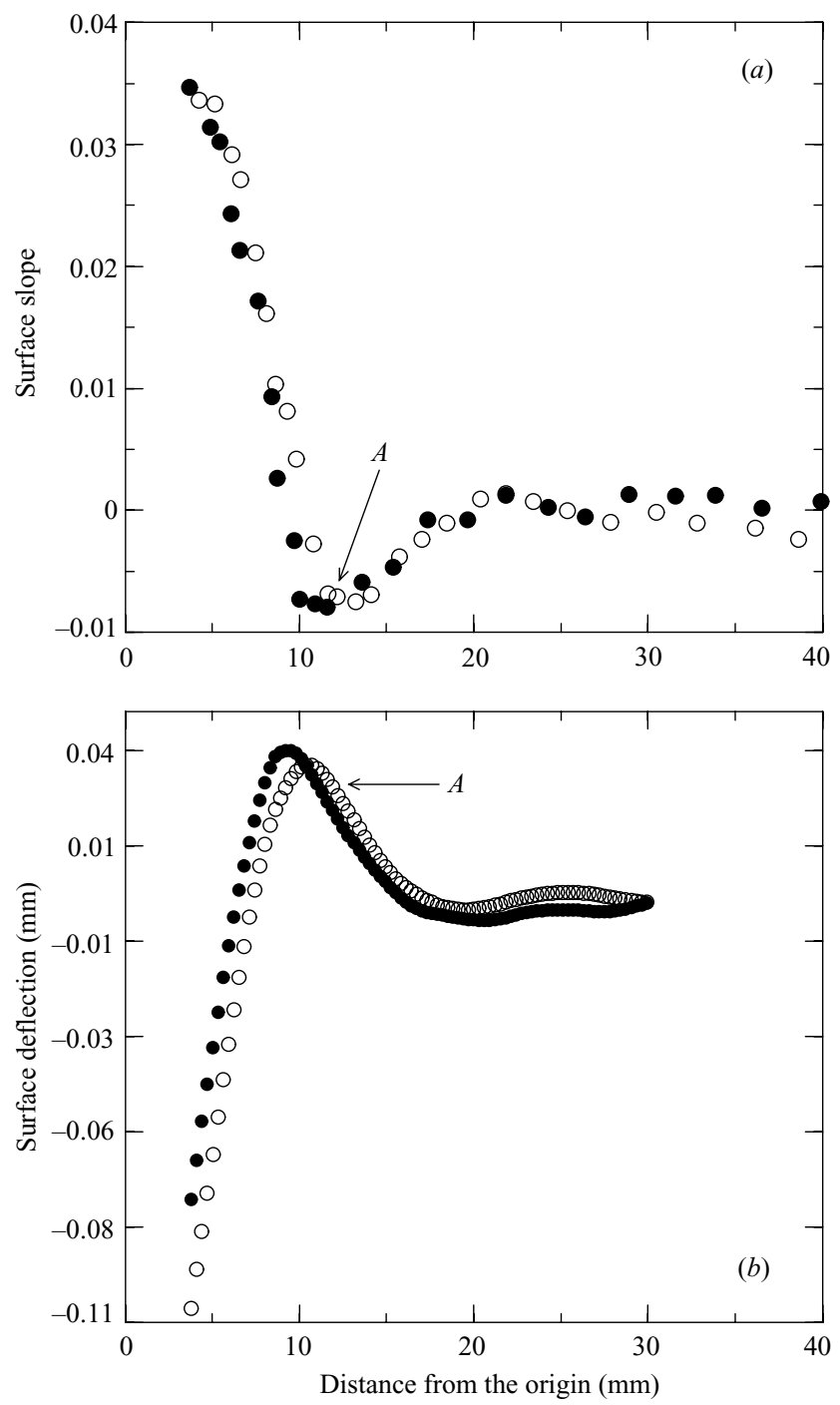

FiguRE 4. (a) Measured surface slope $0.3 \mathrm{~s}$ after deposition. (b) Interface profile obtained by integration. Moiré data from the right- (open circles) and left-hand (filled circles) side of drop used in reconstructing slope and interface profile. Location of slope minimum designated by $A$.

\subsection{Spreading exponents}

The radial distance between the point of deposition and the position of the slope minimum $A$, called $R_{\text {min }}$, was used to track the advancing rim of the oleic acid film. The distance between the point of deposition and the position of the maximum slope $B$, called $R_{\max }$, indicates the growth of the very thin region formed around the deposited droplet of oleic acid. For the experiments in which the liquid surface was covered with talc particles and simultaneously observed with the Moire apparatus, it was noted that the Moire fringes became distorted long before any talc front appeared. The talc particles, which were readily pushed aside by the advancing oleic acid, had to aggregate to a sizeable number to produce a visible receding front. By the time the talc front was visible, the slope of the advancing rim had decreased to a point where 


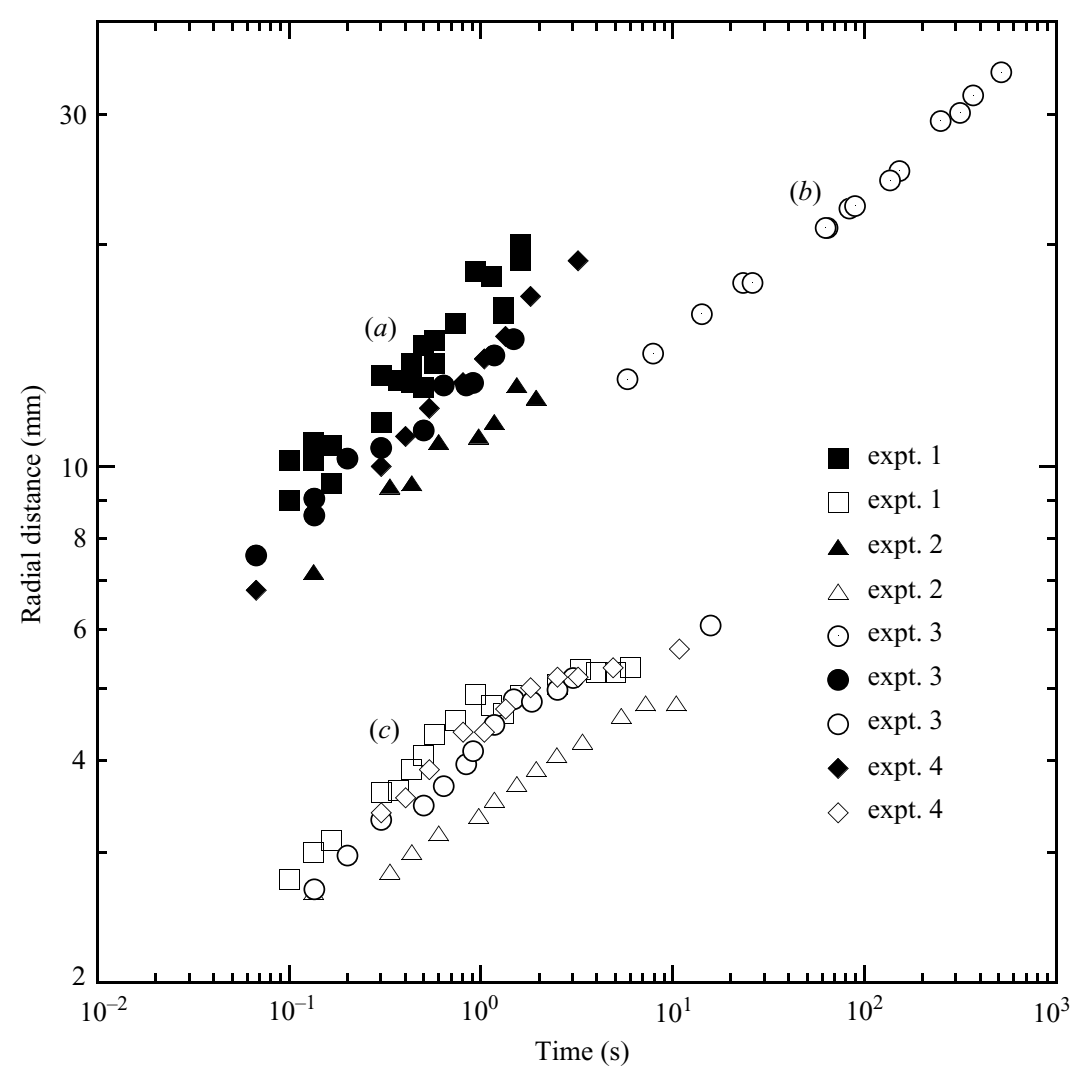

FIGURE 5. Propagation of three positions along spreading film: (a) slope minimum, (b) talc front and (c) slope maximum.

the distortion in the fringes was no longer measurable. These two measurements somewhat complemented each other in this respect since the front could be tracked by two different means over an extended period of time. We did not use talc particles in most of our runs, however, because of the contaminant issue raised by introducing foreign agents on the liquid surface. Systematic tracking of the slope minimum and maximum (for $0.7 \mathrm{~s} \leqslant t \leqslant 3.0 \mathrm{~s}$ ) as well as the talc front (for $5.8 \mathrm{~s} \leqslant t \leqslant 500 \mathrm{~s}$ ), as plotted in figure 5, revealed that within each run each of these quantities could be fitted to a power law of the form $R_{i}=K_{i} t^{a_{i}}$ where $i=\min$ (slope minimum), $t$ (talc front), or max (slope maximum). The data, which was fitted using a least-squares routine in which the coefficients, $K_{i}$, and the exponents, $a_{i}$ were adjustable parameters, is summarized in table 1 . For experiment 1 , the data obtained from the left and right of the spreading drop is presented separately to show the degree of variation within a single run. In experiment 3 , the drop was deposited off-centre in order to visualize simultaneously the presence of a talc front. Only the right-hand side fringe could be analysed.

The average value of the spreading exponent for the advancing rim was found to be $a_{\min }=0.23 \pm 0.03$ (table 1 , column 4). The value of $a_{\min }$ is close to the theoretical value of $1 / 4$ which has been derived for the case of an insoluble surfactant monolayer spreading from a finite source in axisymmetric geometry when hydrostatic, capillary and surface diffusion effects are negligible in comparison to the Marangoni effect (Jensen \& Grotberg 1992; Espinosa et al. 1993). The theoretical argument leading 


\begin{tabular}{|c|c|c|c|c|c|c|c|c|c|c|}
\hline Exp. & $V(\mu 1)$ & $K_{\min }$ & $a_{\min }$ & $r_{\min }$ & $K_{t}$ & $a_{t}$ & $r_{t}$ & $K_{\max }$ & $a_{\max }$ & $r_{\max }$ \\
\hline 1 (left) & 0.25 & $16.64 \pm 0.30$ & $0.26 \pm 0.02$ & 0.972 & $\cdots$ & $\cdots$ & $\cdots$ & & $\cdots$ & \\
\hline 1 (right) & 0.25 & $17.11 \pm 0.23$ & $0.23 \pm 0.01$ & 0.980 & $\cdots$ & $\cdots$ & $\cdots$ & $4.40 \pm 0.07$ & $0.15 \pm 0.01$ & 0.950 \\
\hline 2 & 0.25 & $11.33 \pm 0.17$ & $0.20 \pm 0.02$ & 0.973 & $\cdots$ & $\cdots$ & $\cdots$ & $3.48 \pm 0.03$ & $0.15 \pm 0.01$ & 0.992 \\
\hline 3 & 0.50 & $13.61 \pm 0.16$ & $0.21 \pm 0.01$ & 0.989 & $8.81 \pm 0.23$ & $0.21 \pm 0.01$ & 0.997 & $4.14 \pm 0.07$ & $0.16 \pm 0.01$ & 0.967 \\
\hline 4 & 0.50 & $14.04 \pm 0.08$ & $0.27 \pm 0.01$ & 0.998 & $\cdots$ & $\cdots$ & $\cdots$ & $4.37 \pm 0.05$ & $0.18 \pm 0.01$ & 0.980 \\
\hline Avg. & $\cdots$ & $14.55 \pm 0.19$ & $0.23 \pm 0.01$ & $\cdots$ & $8.81 \pm 0.23$ & $0.21 \pm 0.01$ & $\cdots$ & $4.11 \pm 0.06$ & $0.14 \pm 0.01$ & $\cdots$ \\
\hline \multicolumn{11}{|c|}{$\begin{array}{l}\text { TABLE 1. Least-squares fit to experimental data of the form } R_{i}=K_{i} t^{a_{i}} \text {, where } i=\text { min (slope minimum), } t \text { (talc front) or max (slope maximum). } \\
r_{i} \text { denote linear correlation coefficients. Droplet volume denoted by } V \text {. Vertical grating separation, } d \text {, for experiments } 1-3 \mathrm{was} 2 \mathrm{~cm} \text { and for } \\
\text { experiment } 4 \text { was } 7 \mathrm{~cm} \text {. }\end{array}$} \\
\hline
\end{tabular}


to the $1 / 4$ exponent is outlined in the Appendix B. Were the surfactant monolayer supplied instead from an infinite source (i.e. a source at constant concentration), the spreading exponent would have ranged between 1/4 and 1/2 (Jensen \& Grotberg 1992; Espinosa et al. 1993). It is perhaps surprising that the advancing front behaves as if it were fed from a finite source whose concentration is depleting in time since the initial deposited mass of oleic acid greatly exceeds the amount of oleic acid needed to cover the entire surface with a saturated monolayer. The total mass of oleic acid contained in the droplet volume deposited on the surface $(V=0.5 \mu \mathrm{l})$ is $0.44 \mathrm{mg}$. Using the isotherm of adsorption of oleic acid on pure water, the surface concentration corresponding to saturation $\left(\Pi=22 \mathrm{mN} \mathrm{m}^{-1}\right)$ is found to be $1.53 \times 10^{-7} \mathrm{~g} \mathrm{~cm}^{-2}$. As the total surface area to be covered is $284 \mathrm{~cm}^{2}$, the mass of oleic acid necessary to cover the entire surface with the saturated monolayer is $0.043 \mathrm{mg}$ which represents only $10 \%$ of the total mass of oleic acid deposited on the surface. In the presence of glycerol, the oleic monolayer is expected to be more expanded (Lim \& Berg 1975), that should reduce even more the amount of oleic acid necessary to cover the surface for the same surface pressure $\left(\Pi=22 \mathrm{~m} \mathrm{~m}^{-1}\right)$. However, the visualization from the side indicates that most of the oleic acid remains at the point of deposition. We believe that the drastic thinning around the droplet occurring early in the experiment $(t=0.3 \mathrm{~s})$ may be slowing or halting the supply from the source to the spreading film. It is uncertain whether or not the thinning process would lead to film rupture near the point of original deposition. It was observed, however, that during the course of the experiment the region around the droplet could not be rewetted by the glycerol solution. This seems to suggest that rupture of the aqueous film may have taken place.

The experimental values of the coefficient $K_{\min }$ (table 1, column 3) of the power law for the advancing front cannot be easily compared with the theoretical expression $K=c\left(4 \beta M^{*} H_{o}^{*} / \mu^{*}\right)^{1 / 4}$ given in the Appendix because $c$ is an undetermined constant and $\beta=\partial \sigma^{*} / \partial \Gamma^{*}$ is unknown for our system. However, it is likely that the variations between the experimental values of $K_{\min }$ for the different runs are related to the fact that the mass $M^{*}$ of oleic acid involved in the spreading can vary from experiment to experiment depending on how and when the supply from the source (droplet) has been halted. The talc front propagation fitted a power law with an exponent $a_{t}$ very close to $a_{\min }$ (table 1 , columns 4 and 7) but the coefficient $K_{t}$ is significantly smaller than $K_{\min }$ (table 1, columns 3 and 7). This indicates that the talc is pushed by the monolayer of oleic acid and that the peak of the advancing rim is ahead of the monolayer. We found that the thin region grows slowly in time (3 to 4 times slower than the advancing rim) and that the growth of this region seems to follow a power law with an exponent $a_{\max }=0.16 \pm 0.01$ (table 1, column 10).

\section{Theoretical formulation}

Since the exponent found experimentally for the spreading law so closely matches the theoretical exponent for a finite source, we employ a lubrication-type model which is relevant for a finite source (Jensen \& Grotberg 1992; Grotberg, Halpern \& Jensen 1995) of insoluble surfactant. The model assumes that the surfactant is delivered as a surface patch of surfactant monolayer of prescribed concentration distribution whereas, experimentally, the surfactant is delivered in the form of a droplet. In addition, the model uses a linear equation of state to describe the dependence of the surface tension of the glycerol-water layer on the surface concentration of oleic acid, which is an approximation of that used for oleic acid-water systems by TomoaiaCotisel et al. (1987). The goal of this study, is, therefore, to determine whether, in spite 

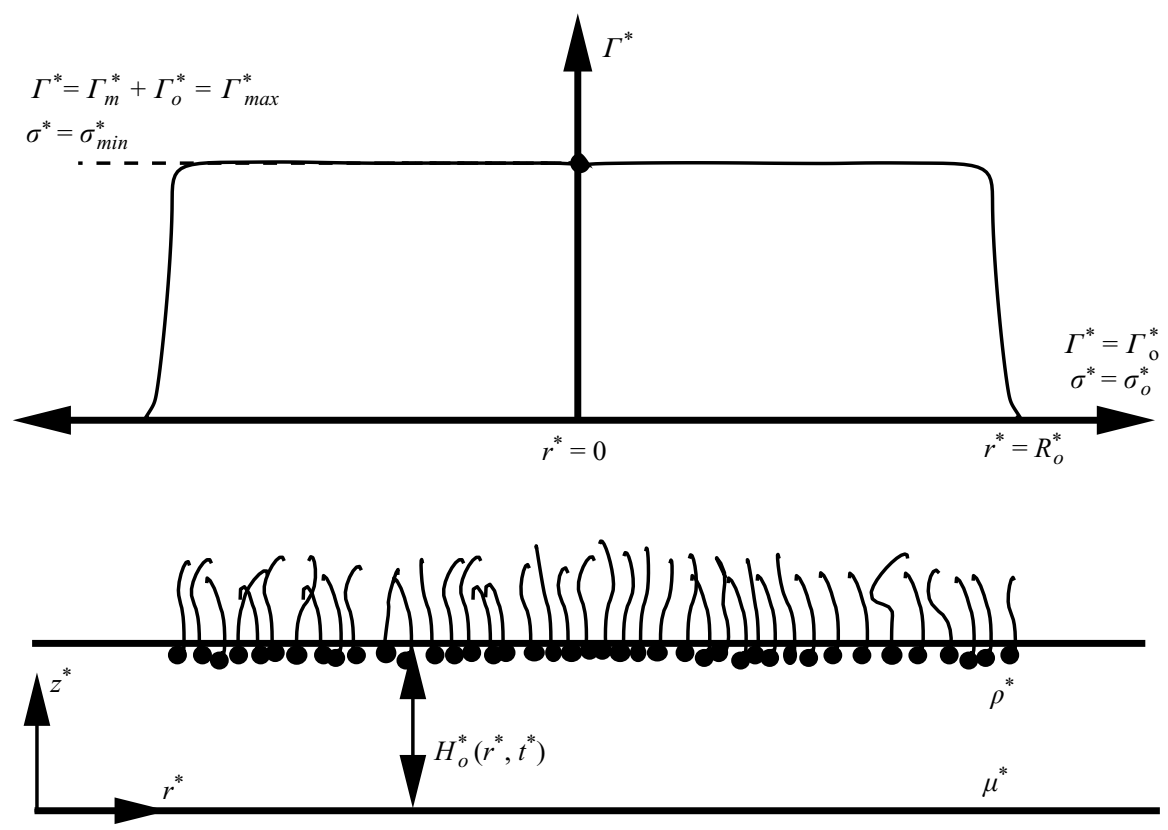

FIGURE 6. Initial monolayer distribution on a thin flat liquid layer. $\Gamma_{o}^{*} \neq 0$ represents pre-existing surface contamination where $\Gamma_{o}^{*} \ll \Gamma_{\max }^{*}$.

of these simplifications, the model is able to provide a reasonable description of the surface shape and its time evolution by adjusting the initial conditions appropriately.

The initial film of glycerol solution present before deposition of the oleic acid droplet is modelled by an incompressible Newtonian film of initial uniform thickness $H_{o}^{*}$, viscosity $\mu^{*}$ and density $\rho^{*}$, supported by a smooth flat substrate at $z^{*}=0$. As we suspected, the glycerol solution was contaminated, based on the measurement of surface pressure, the initial film of glycerol solution will be considered first free of contaminant and then with a small uniform concentration of pre-existing surfaceactive contaminant, $\Gamma_{o}^{*}$. A surface patch of radius $R_{o}^{*}$ containing an oleic acid monolayer of uniform concentration $\Gamma_{m}^{*}$ is delivered to the quiescent liquid film of glycerol solution (see figure 6). Initially, the total surfactant concentration resembles a top-hat function with maximum value, $\Gamma_{\max }^{*}=\Gamma_{m}^{*}+\Gamma_{o}^{*}$, which matches smoothly onto the background concentration, $\Gamma_{o}^{*}$, near the radial coordinate $r^{*}=R_{o}^{*}$. The extent of the radius $R_{o}^{*}$, the concentration $\Gamma_{m}^{*}$ and the initial surfactant distribution control the finite mass of oleic acid which spreads on the surface. For our comparison, they represent parameters which are unknown and have to be chosen in order to simulate the real spreading process. The surface tension $\sigma_{\text {min }}^{*}$ denotes the minimum of surface tension corresponding to the maximum of total surface concentration $\Gamma_{\max }^{*}$ whereas $\sigma_{o}^{*}$ is the surface tension of the glycerol solution before deposition of oleic acid. The spreading surface pressure is defined as $\Pi^{*}=\sigma_{o}^{*}-\sigma_{\min }^{*}$.

The equations of motion for the film thickness and total surfactant concentration are derived in the lubrication approximation for which $\varepsilon=H_{o}^{*} / R_{o}^{*} \ll 1$. The monolayer and underlying fluid spread spontaneously toward the regions of smallest surface concentration under the action of an initial gradient in surface tension which scales as $\Pi^{*} / R_{o}^{*}$. This flow is counterbalanced by the viscous stress at the surface which is of order $\mu^{*} U^{*} / H_{o}^{*}$. The spreading velocity characteristic of Marangoni-driven flow 
in thin films is determined from this tangential stress balance to be $U^{*}=\varepsilon \Pi^{*} / \mu^{*}$. The lubrication equations are reduced to dimensionless form by introducing several characteristic scales according to the following: the radial and vertical coordinates are $r=r^{*}$ / $R_{o}^{*}, z=z^{*} / H_{o}^{*}$, the radial and vertical velocities are $u=u^{*} / U^{*}, v=v^{*} / \varepsilon U^{*}$, and the dimensionless time and pressure are defined by $\tau=t^{*} U^{*} / R_{o}^{*}$ and $p=p^{*} H_{o}^{* 2} / \mu^{*} U^{*} R_{o}^{*}=$ $p^{*} H_{o}^{*} / \Pi^{*}$. The local surface concentration denoted by $\Gamma^{*}\left(r^{*}, t^{*}\right)=\Gamma_{1}^{*}\left(r^{*}, t^{*}\right)+$ $\Gamma_{2}^{*}\left(r^{*}, t^{*}\right)$, where $\Gamma_{1}^{*}$ denotes the concentration of oleic acid and $\Gamma_{2}^{*}$ denotes the concentration of the surface active contaminant (Grotberg et al. 1995), is normalized by $\Gamma_{\max }^{*}=\Gamma_{m}^{*}+\Gamma_{o}^{*}$. The local surface tension is related to the local surface concentration through the equation of state. We choose the following linear equation of state: $\sigma^{*}=\sigma_{o}^{*}-\beta \Gamma^{*}$ where $\beta=\partial \sigma^{*} / \partial \Gamma^{*}$ is a constant (Tomoaia-Cotisel et al. 1987). The local surface tension is normalized by $\sigma=\left(\sigma^{*}-\sigma_{\text {min }}^{*}\right) /\left(\sigma_{o}-\sigma_{\text {min }}^{*}\right)$ in order to rewrite the equation of state as $\sigma=1-\Gamma$.

The computations are performed in a stretched coordinate system in order to maximize computational efficiency (Jensen \& Grotberg 1992):

$$
\xi=\frac{r}{\tau^{a}}, \quad H(r, \tau)=h(\xi, \tau), \quad \Gamma(r, \tau)=\frac{g(\xi, \tau)}{\tau^{b}}=\frac{g_{1}(\xi, \tau)+g_{2}(\xi, \tau)}{\tau^{b}},
$$

where the exponents $a$ and $b$ relevant to the axisymmetric spreading of a finite amount of surfactant on a thin liquid film are $a=1 / 4$ and $b=1 / 2$ (Jensen \& Grotberg 1992; Espinosa et al. 1993). The coupled system of evolution equations governing the spreading dynamics are then given by

$$
\begin{aligned}
\tau h_{\tau}= & a \xi h_{\xi}+\frac{1}{2} \partial_{\xi}\left(h^{2} g_{\xi}\right)+\left(\frac{1}{3} B o \tau^{b}\right) \partial_{\xi}\left(h^{3} h_{\xi}\right)-\left(\frac{\mathscr{C}}{3 \tau^{4 a-1}}\right) \partial_{\xi}\left(h^{3}\left[\partial_{\xi}\left(h_{\xi}\right)\right]_{\xi}\right), \\
\tau g_{\tau}= & b g+a \xi g_{\xi}+\partial_{\xi}\left(g h g_{\xi}\right)+\left(\frac{1}{2} B o \tau^{b}\right) \partial_{\xi}\left(g h^{2} h_{\xi}\right) \\
& +\left(\frac{\tau^{b}}{P e_{s}}\right) \partial_{\xi}\left(g_{\xi}\right)-\left(\frac{\mathscr{C}}{2 \tau^{4 a-1}}\right) \partial_{\xi}\left(g h^{2}\left[\partial_{\xi}\left(h_{\xi}\right)\right]_{\xi}\right), \\
\tau g_{1_{\tau}}= & b g_{1}+a \xi g_{1_{\xi}}+\partial_{\xi}\left(g_{1} h g_{\xi}\right)+\left(\frac{1}{2} B o \tau^{b}\right) \partial_{\xi}\left(g_{1} h^{2} h_{\xi}\right) \\
& +\left(\frac{\tau^{b}}{P e_{s}}\right) \partial_{\xi}\left(g_{1_{\xi}}\right)-\left(\frac{\mathscr{C}}{2 \tau^{4 a-1}}\right) \partial_{\xi}\left(g_{1} h^{2}\left[\partial_{\xi}\left(h_{\xi}\right)\right]_{\xi}\right) .
\end{aligned}
$$

Here, $\mathscr{C} \equiv \varepsilon^{2} \sigma_{\text {min }}^{*} / \Pi^{*}$, a capillary parameter, $P e_{s} \equiv U^{*} R_{o}^{*} / \mathscr{D}_{s}^{*}=\Pi^{*} H_{o}^{*} / \mu^{*} \mathscr{D}_{s}^{*}$, is a surface Péclet number, $P e_{s} \equiv U^{*} R_{o}^{*} / \mathscr{D}_{s}^{*}=\Pi^{*} H_{o}^{*} / \mu^{*} \mathscr{D}_{s}^{*}$, and $B o \equiv \rho^{*} g^{*} H_{o}^{* 2} / \Pi^{*}$, a Bond number. The solution for the endogenous concentration field, $g_{2}(\xi, \tau)$, can be computed directly from the equality $g_{2}=g-g_{1}$.

The initial conditions at $\tau=\tau_{o}$ are given by

$$
\begin{aligned}
h\left(\xi, \tau_{o}\right) & =1, \\
g_{1}\left(\xi, \tau_{o}\right) & =0.5\left(A-g_{o}\right)\left[1-\tanh \left(B\left(\xi-\xi_{o}\right)\right)\right], \\
g_{2}\left(\xi, \tau_{o}\right) & =g_{o}, \\
g\left(\xi, \tau_{o}\right) & =0.5\left(A-g_{o}\right)\left[1-\tanh \left(B\left(\xi-\xi_{o}\right)\right)\right]+g_{o} .
\end{aligned}
$$

These choices represent a typical circular patch of insoluble surfactant spreading onto a flat uniform film containing a very small level of pre-existing surface-active contaminant. Figure 7 depicts the surface concentration fields for parameter values $A=1$, $B=50, \xi_{o}=1.0$ (which were used in all the computations) and $g_{o}=0.0291$. The following boundary conditions enforce the constraint of no flux of fluid and surfactant at 


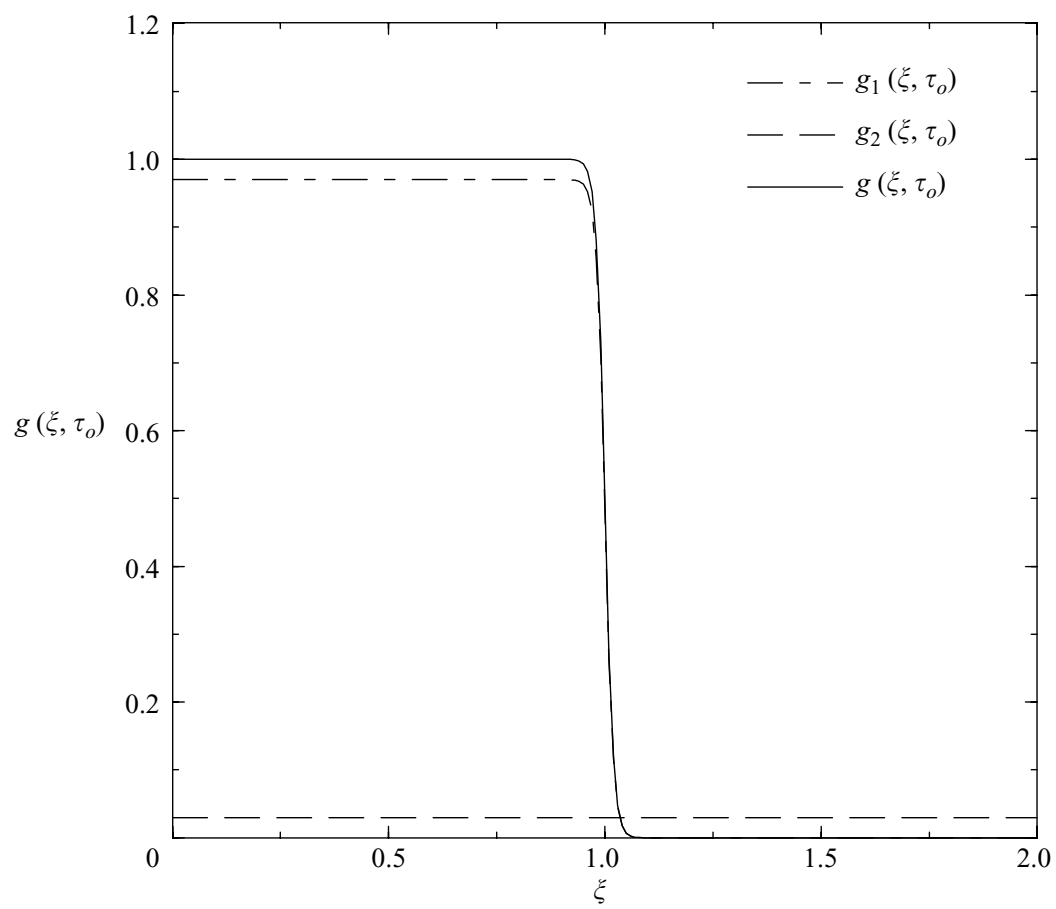

FIGURE 7. Initial condition of surfactant concentrations for $A=1, B=50, \xi_{o}=1.0$ and $g_{o}=0.0291$. Exogenous species $=g_{1}\left(\xi, \tau_{o}\right)$, endogenous species $=g_{2}\left(\xi, \tau_{o}\right)$, where $g=g_{1}+g_{2}$.

the origin and recovery of quiescent conditions downstream:

$$
\begin{array}{rlrl}
h_{\xi}(0, \tau) & =0, & h_{\xi \xi \xi}(0, \tau) & =0, \\
h(\infty, \tau)=1, & h_{\xi}(\infty, \tau) & =0, \\
g_{\xi}(0, \tau)=0, \quad g_{1_{\xi}}(0, \tau)=0, & g_{2_{\xi}}(0, \tau) & =0, \\
g(\infty, \tau)=g_{o} \tau^{b}, & g_{1}(\infty, \tau)=0, & g_{2}(\infty, \tau) & =g_{o} \tau^{b} .
\end{array}
$$

Numerical solutions of the evolution equations were obtained using the method of lines (Schiesser 1991) using fourth-order centred differences to discretize spatial derivatives and Gear's method to advance the solution in time. The number of grid points used ranged from 300 to 951 ; convergence was achieved upon mesh refinement. The spatial locations of the minimal surface slope and monolayer leading edge, $R_{\min }$ and $R_{t}$, respectively, were also determined numerically, the latter defined as follows:

$$
0.99 M=2 \pi \int_{0}^{R_{t}} \xi g_{1}(\xi, \tau) \mathrm{d} \xi,
$$

where $M$ is the total mass of deposited surfactant.

\section{Comparison between numerical and experimental results}

The parameters $\mathscr{C}, P e_{s}$ and $B o$ depend on unknown quantities, the surface diffusivity of the oleic acid on the glycerol solution, the radial extent of the initially deposited monolayer, $R_{o}^{*}$, and the surface spreading pressure, $\Pi^{*}$. In all the computations, the surface diffusivity was fixed and assumed to be $10^{-5} \mathrm{~cm}^{2} \mathrm{~s}^{-1}$. Because the value of $P e_{s}$ 


\begin{tabular}{ccccccccc}
\hline Case $\Pi^{*}$ & $\left(\mathrm{dyn} \mathrm{cm}^{-1}\right)$ & $R_{o}^{*}(\mathrm{~cm})$ & $g_{o}$ & $\varepsilon$ & $P e_{s}\left(10^{5}\right)$ & $\mathscr{C}$ & $B o$ & $\varepsilon R e$ \\
1A & 22 & 0.25 & 0 & 0.10 & 5.16 & 0.018 & 0.032 & 0.56 \\
1B & 22 & 0.25 & 0.0291 & 0.10 & 5.16 & 0.018 & 0.032 & 0.56 \\
3A & 22 & 0.18 & 0.0196 & 0.14 & 5.16 & 0.035 & 0.032 & 1.09 \\
3B & 22 & 0.20 & 0.0196 & 0.13 & 5.16 & 0.028 & 0.032 & 0.87 \\
3C & 10 & 0.21 & 0.0196 & 0.12 & 2.35 & 0.073 & 0.071 & 0.36
\end{tabular}

TABLE 2. Parameter values used in computation of surface slope and surface interface profiles for experiments 1 and 3. Here, $R e \equiv\left(\rho^{*} U^{*} H_{o}^{*}\right) / \mu^{*}=\left(\varepsilon^{2} \rho^{*} \Pi^{*} R_{o}^{*}\right) / \mu^{* 2}$ represents the Reynolds number.

is very large, a variation of the surface diffusivity by one or two orders of magnitude does not have a significant effect on the result. The six parameters that remain to be specified for the computation are: $R_{o}^{*}, \Pi^{*}, A, B, \xi_{o}$ and $g_{o}$. The radial extent of the initially deposited monolayer, $R_{o}^{*}$, depends on the method used to deliver the surfactant material. Whereas Gaver \& Grotberg (1992) took $R_{o}^{*}$ to be the radius of the restraining ring, our experiments on much thinner films precluded the use of this convenient technique. We therefore treated $R_{o}^{*}$ as an adjustable parameter in our numerical work. The other variable required for computing the film thickness and concentration profiles is the total mass of surfactant delivered to the liquid layer. As outlined in the Appendix, the mass is controlled by the product $\Pi^{*} R_{o}^{* 2}$. In trying to use parameter values consistent with the experiments performed, $\Pi^{*}$ was fixed at a value of $22 \mathrm{dyn} \mathrm{cm}^{-1}$ (as found in $\S 2.1$ ) and $R_{o}^{*}$ was varied, or else $R_{o}^{*}$ was held fixed and $\Pi^{*}$ was varied. A complete optimization algorithm was not implemented in fitting the numerical solutions to the experimental data. Instead, simple visual inspection was used to bring the numerical data in line with the experimental measurements. As evident, however, the final parameter values cited generated strong agreement between the numerical and experimental values for both the interface slope and film thickness, particularly in the vicinity of the advancing rim, as well as the film spreading rates. Hereinafter, we refer to the parameters determined in this visual way as the 'fitted' parameters, with the understanding that these have not been obtained by any optimization technique.

In table 2, we give the fitted parameter values for the cases which yielded very good agreement with the experimental data (1B and $3 \mathrm{C}$ ) via visual inspection, as well as a few examples to illustrate the effects on the spreading process caused by $\Pi^{*}$ and $R_{o}^{*}$. The visually obtained closest fits to the experimental data for runs 1 and 3 (cases $1 \mathrm{~B}$ and $3 \mathrm{C}$ described below) were achieved with $R_{o}^{*}=2.5 \mathrm{~mm}$ and $2.1 \mathrm{~mm}$. This length proved to be approximately four times the radius of the droplet when suspended above the liquid layer in figure $1(a)$ estimates for $R_{o}^{*}$ and $\Pi^{*}$ which would yield values for $R_{\min }$ consistent with experiment. Cases $1 \mathrm{a}\left(g_{o}=0\right)$ and $1 \mathrm{~B}\left(g_{o}=0.03\right)$ illustrate the effect of pre-existing surface contamination of the liquid layer. A good fit to the experimental surface slope, the spreading coefficient, and spreading exponent was not obtained unless $g_{o} \neq 0$. Cases $3 \mathrm{~A}$ and $3 \mathrm{~B}$ demonstrate the effect of increasing the total mass of available surfactant, while $3 \mathrm{~B}$ and $3 \mathrm{C}$ illustrate the effect caused by lowering the spreading pressure $\Pi^{*}$. In what follows, we directly compare the numerical results for the evolution of the surface slope, the interface profile, and the spreading coefficients and exponents with the same quantities obtained experimentally. 


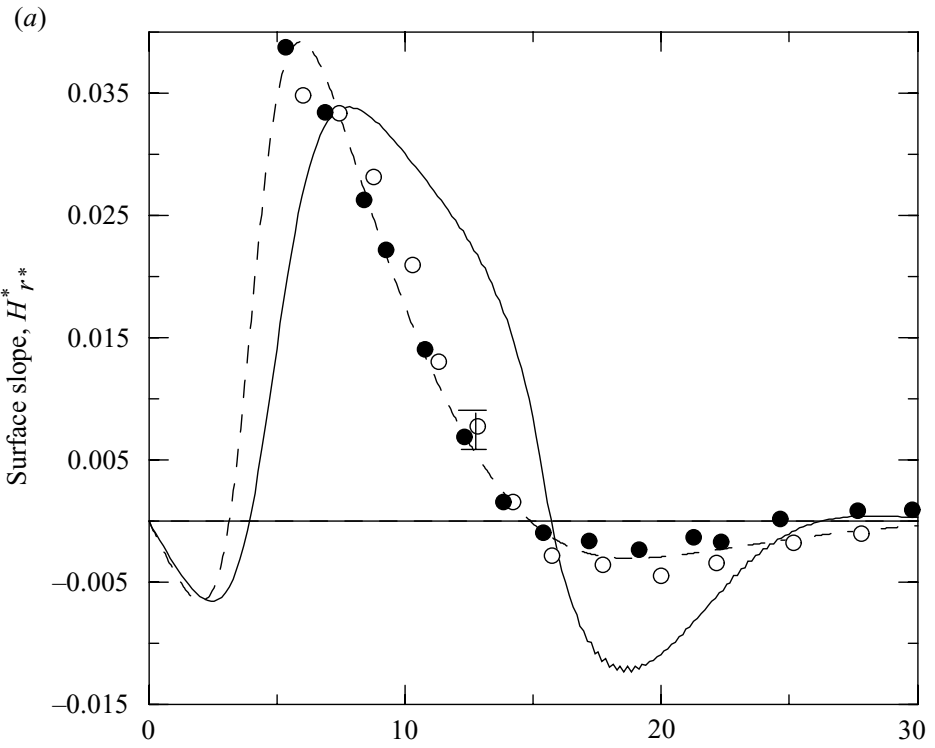

(b)

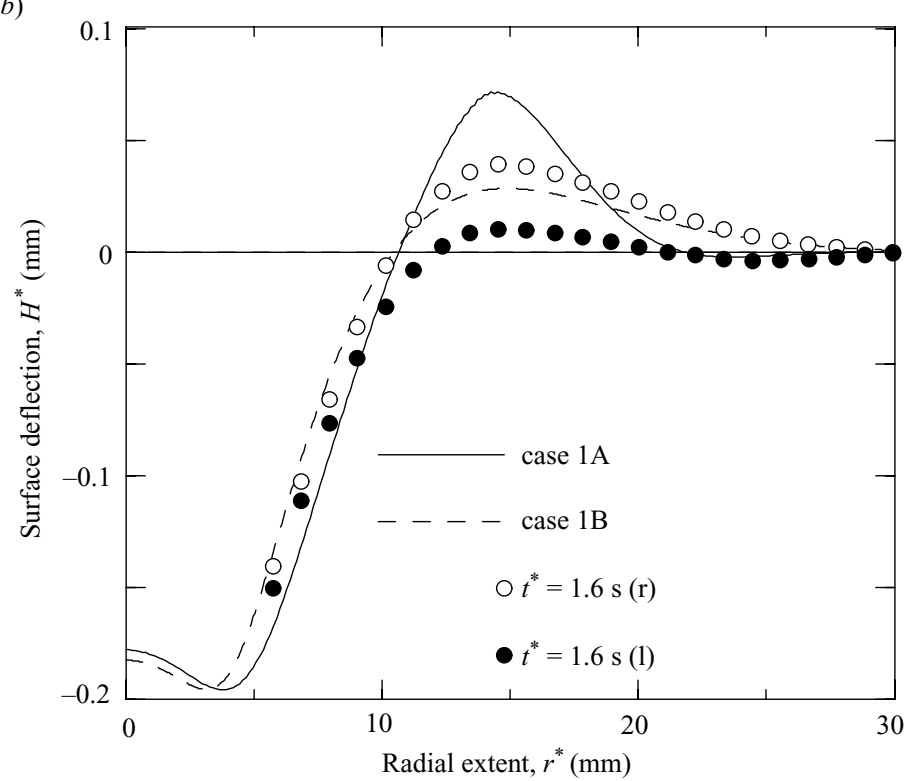

FigURE 8. Experimental (right (r) and left (1) Moire fringe) and theoretical comparison of surface slope and interface profiles from experiment 1 at $t^{*}=1.6 \mathrm{~s}$ after deposition. Parameter values for cases $1 \mathrm{~A}$ and $1 \mathrm{~B}$ given in table 2. Experimental error bar shown is the same for all points and caused by the manual tracking of a fringe edge.

\subsection{Surface slope and interfacial profile}

Figure 8 shows data from experiment 1 for the surface slope and deflection profile at time $t^{*}=1.6 \mathrm{~s}$ after deposition plotted against two numerical profiles labelled $1 \mathrm{~A}$ and $1 \mathrm{~B}$. The open and filled circles represent the experimental data obtained from the Moire fringes on the left- and right-hand side of a spreading droplet while the solid and dashed lines represent theoretical curves. The two theoretical profiles underscore the 
(a)
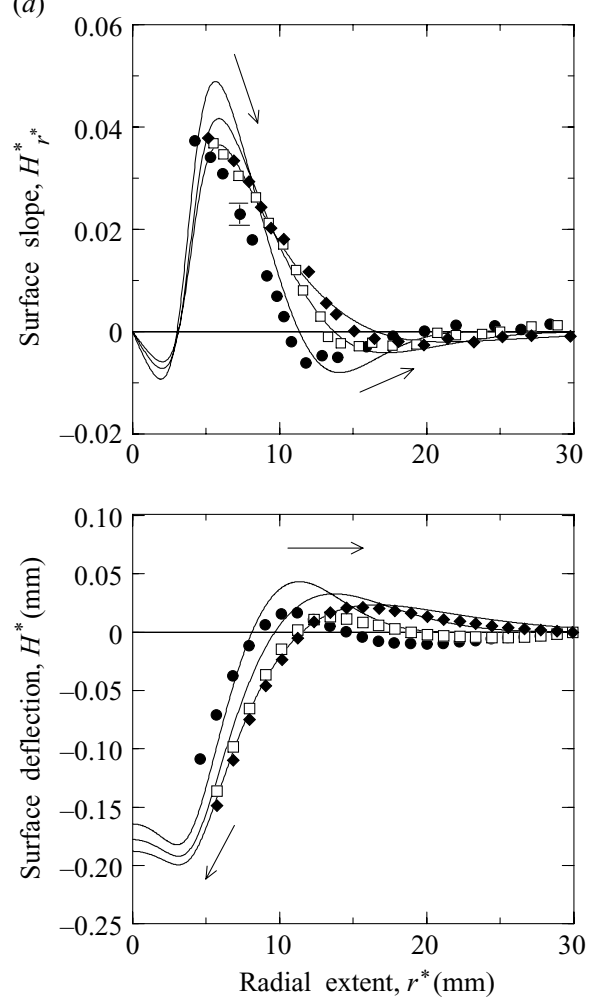

(b)
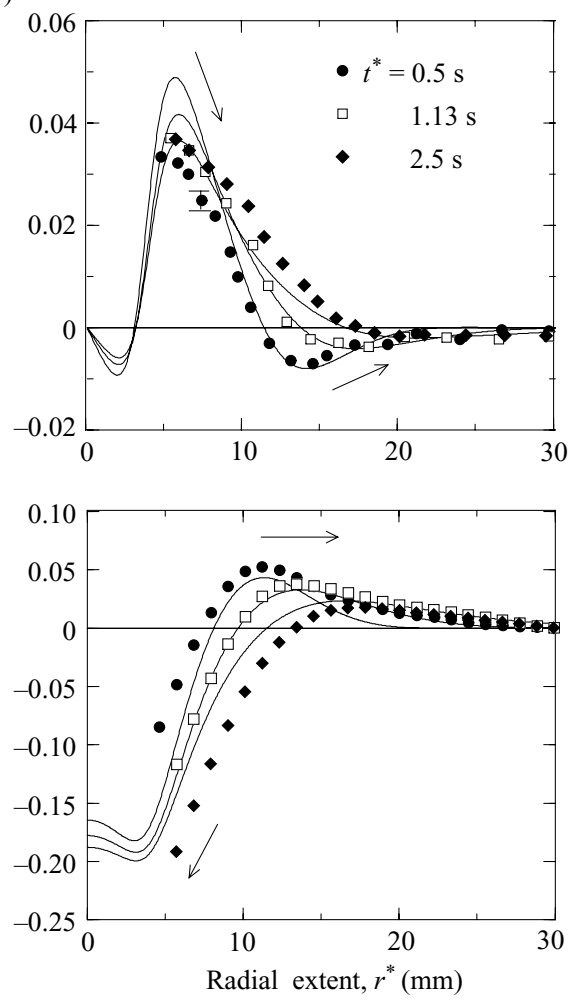

FIGURE 9. Experimental ( $(a)$ left Moiré fringe, $(b)$ right Moiré fringe) and theoretical comparison of the evolution in the surface slope and interface profiles from experiment 1 . Solid lines represent theoretical predictions at time $t^{*}=0.5,1.13$ and $2.5 \mathrm{~s}$ with arrows indicating direction of increasing time. Error bar of same origin as in figure 8.

effect created by introducing a small level of pre-existing contaminant $\left(g_{o}=0.0291\right)$. The curve labelled 1B, describing the surface profile in the presence of endogenous contaminant, closely fits the experimental data. For all the parameters tried, good agreement between theory and experiment always required a small non-zero value of $g_{o}$. This seems to confirm the presence of a small level of surface contamination which was suspected from the experimental measurement of the surface tension of the glycerol-water mixture reported in $\S 2.1$.

The profiles shown in figure 9 describe the evolution of the liquid interface at times $t^{*}=0.50,1.33$ and $2.5 \mathrm{~s}$ after deposition. Figures $9(a)$ and $9(b)$ represent the data obtained from the left- and right-hand sides of the spreading droplet, respectively. The theoretical profiles were computed from the parameter values for case $1 \mathrm{~B}$. The experimental and theoretical profiles agree quite well, not only in the location of the advancing rim as a function of time, but also in the shape and magnitude of the slope and film thickness. Although the model slightly over-predicts the maximum in the slope at the earliest time shown, it rapidly converges toward the experimental data at later times. The overestimate in the initial surface slope is not surprising since the model assumes that the surfactant is delivered instantaneously as a monolayer, while in the experiments, the surfactant is delivered as a droplet whose initial radius exceeds the thickness of the liquid layer. Capillary, hydrostatic and inertial effects due to the 

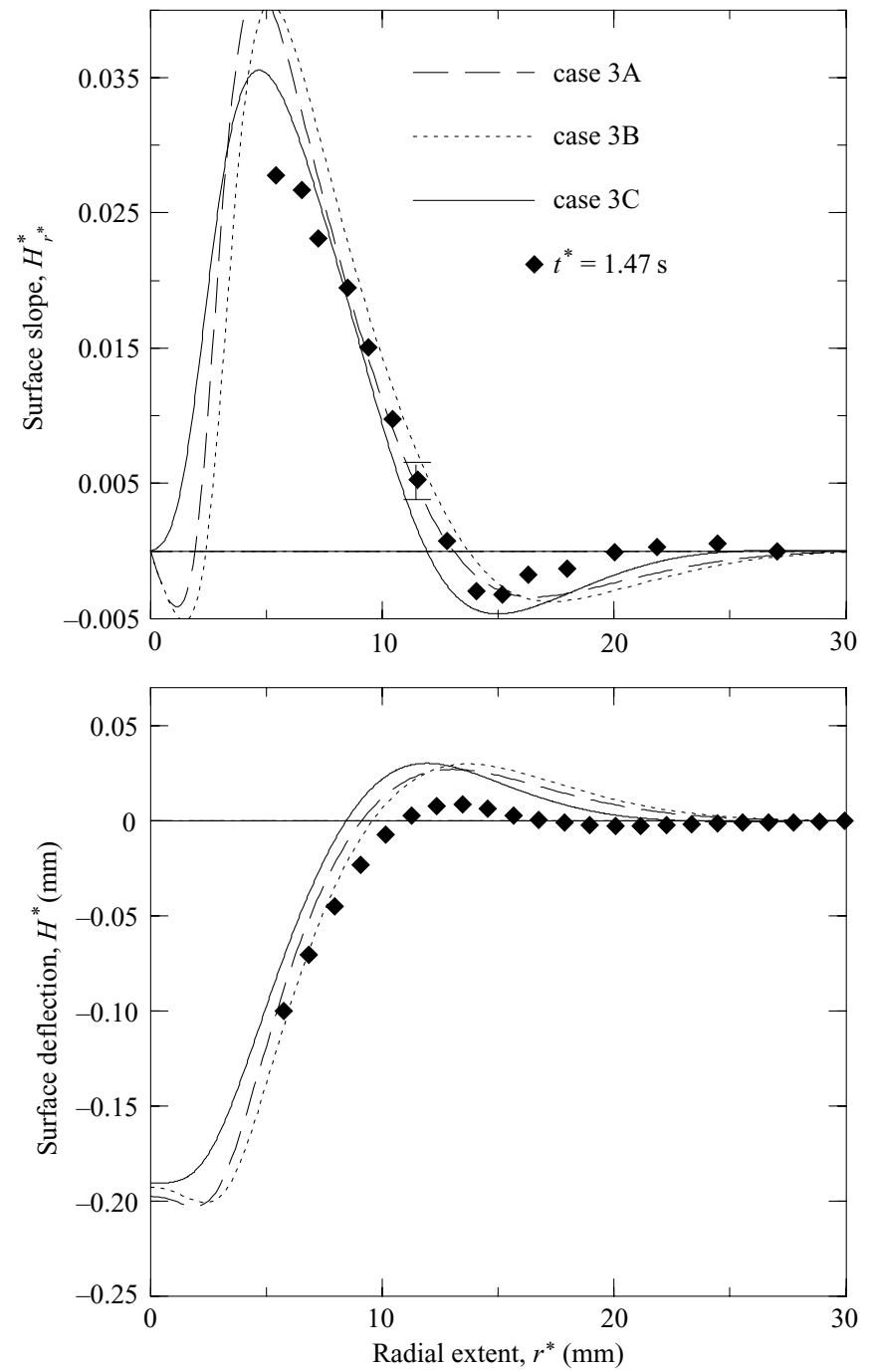

FiguRE 10. Experimental and theoretical comparison of surface slope and interface profiles from experiment 3 at $t^{*}=1.47 \mathrm{~s}$ after deposition. Parameter values for cases $3 \mathrm{~A}, 3 \mathrm{~B}$ and $3 \mathrm{C}$ given in table 2. Error bar of same origin as in figure 8.

deposition procedure are not accurately captured by the model at very early times, immediately following the deposition. The magnitude of the slope near the downstream portion of the film, however, is predicted very well by the model at all times.

Figure 10, showing data from experiment 3 at time $t^{*}=1.47 \mathrm{~s}$ after deposition, provides another example for comparison between theory and experiment. For this particular experimental run, the liquid surface had also been seeded with talc particles and the Moire fringes were obtained along with the position of the receding talc front. The talc front, however, was only visible after a time $t^{*}=5.8 \mathrm{~s}$, during which the ultrafine particles aggregated to a high enough density to be observed by the naked eye. The parameter values for cases $3 \mathrm{~A}, 3 \mathrm{~B}$ and $3 \mathrm{C}$ are given in table 2 . For cases $3 \mathrm{~A}$ and $3 \mathrm{~B}$, the spreading pressure was held fixed at $\Pi^{*}=22 \mathrm{dyn} \mathrm{cm}^{-1}$ while the extent of the initial surfactant patch was increased from $R_{o}^{*}=0.18$ to $0.20 \mathrm{~cm}$. For case 

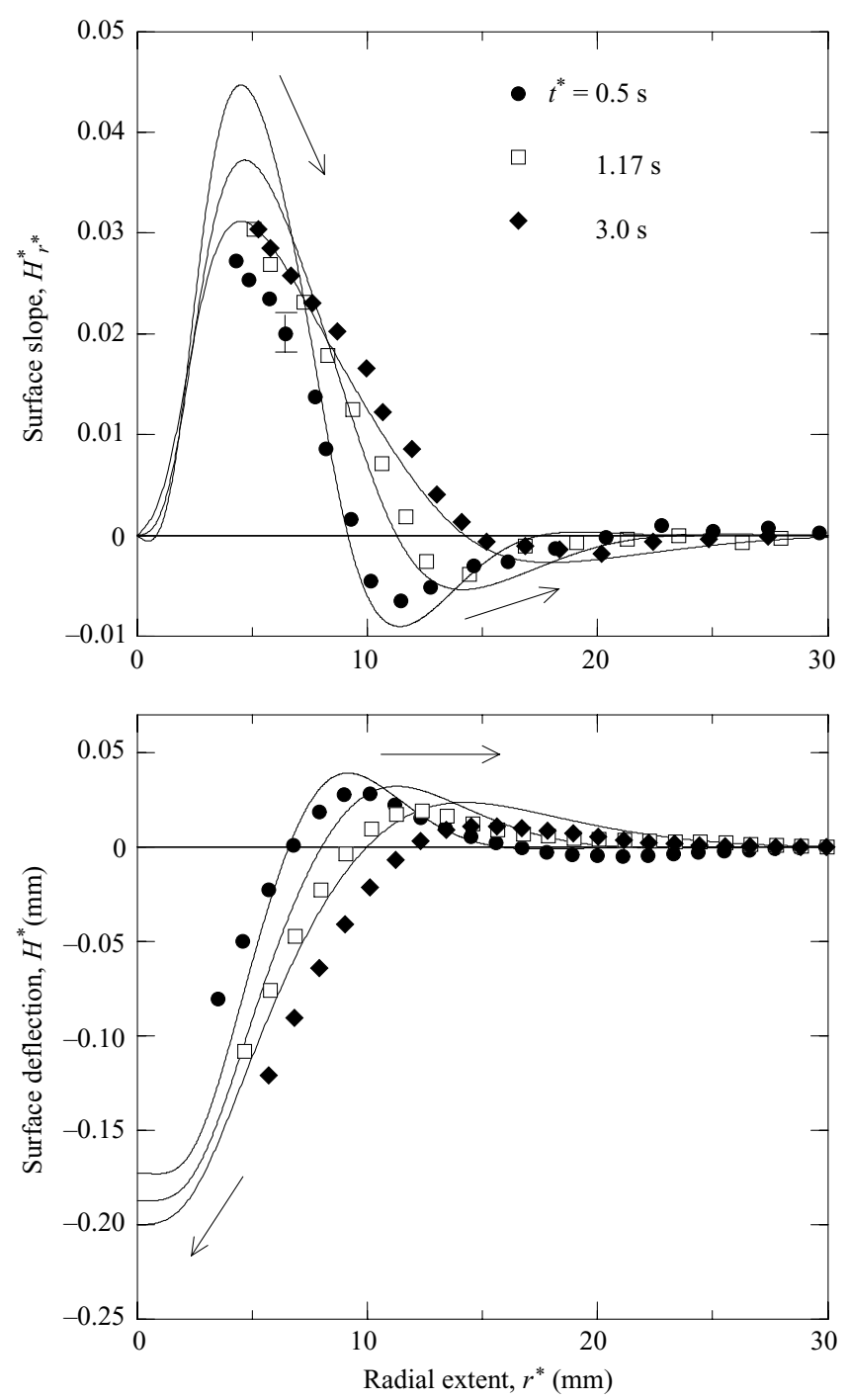

FIGURE 11. Experimental and theoretical comparison of the evolution in the surface slope and interface profiles from experiment 3. Solid lines represent theoretical predictions at time $t^{*}=0.5,1.17$ and $3.0 \mathrm{~s}$ with arrows indicating direction of increasing time. Error bar of same origin as in figure 8.

$3 \mathrm{C}$, the extent of the initial surfactant patch was held at $R_{o}^{*}=0.21 \mathrm{~cm}$, while the spreading pressure was decreased substantially to a value of $\Pi^{*}=10 \mathrm{dyn} \mathrm{cm}^{-1}$. For each parameter set, the theoretical profiles slightly overestimate the amplitude of the maximum slope near the origin and slightly underestimate the slope in the downstream portion. Given the error bar of the experimental slope, each parameter set seems to give a similar reasonable fit to the data. This means that there is not a unique set of parameters $\left(\Pi^{*}, R_{o}^{*}\right)$ which yields reasonable agreement with the experimental data. We found in this case also, that a small but non-zero value of $g_{o}$ was required to produce the film shape and slope observed experimentally. Figure 11 demonstrates the quality of fit in case $3 \mathrm{C}$ as a function of time for $t^{*}=0.50,1.13$ and $2.5 \mathrm{~s}$. Within experimental error, the agreement is once again quite strong. 


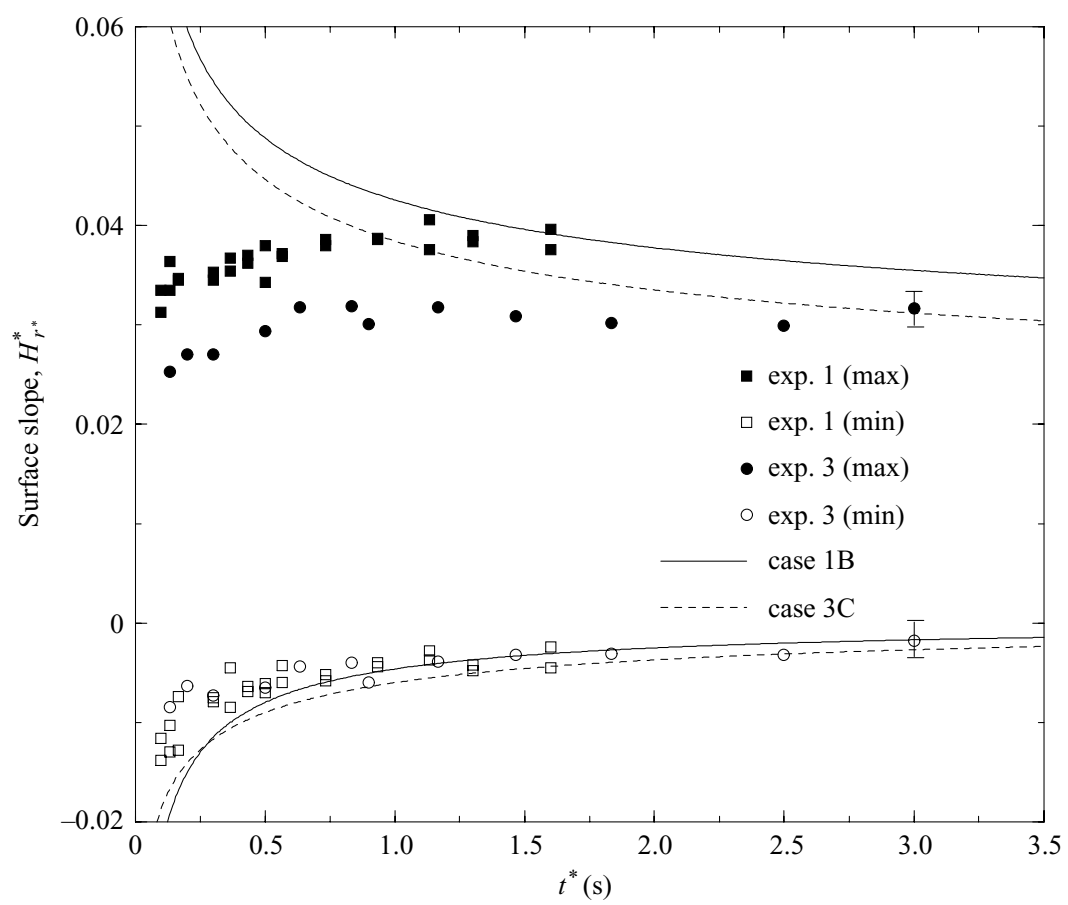

FIGURE 12. Experimental and theoretical comparison of the minimum and maximum value of the surface slope as a function of time. Parameter values for cases $1 \mathrm{~B}$ and $3 \mathrm{C}$ given in table 2.

We combined the results of the numerical data obtained over an interval of $3.0 \mathrm{~s}$ for the magnitude of the maximum and minimum in the slope as a function of time. These data are plotted in figure 12 along with experimental measurements from runs 1 and 3. The minimum in the slope (which occurs downstream of the deposition region) is successfully predicted by the theoretical curves. The maximum in the surface slope is predicted fairly well at late times, but veers away from the experimental data at early times; note that the overall magnitude of the measured surface slopes just exceeds 0.04 , a value small enough for the assumptions of the Moire analysis to be valid, namely $\alpha \ll 1$. The discrepancy between theoretical and experimental maximum slopes at early times may be due to the resolution of the Moire technique employed in the present work not being high enough to provide accurate measurements of the surface slope near the surfactant deposition region. Several other possible reasons for this discrepancy are also described in $\S 6$.

Figure 13 shows the numerically generated surfactant distribution profile for the exogenous and endogenous species and their sum at three different times for parameter values corresponding to $1 \mathrm{~B}$ (figure 13a) and 3C (figure 13b). These profiles were generated with the largest number of grid points used in our computations, $N=951$, in order to resolve the smallest features associated with the junction between $\Gamma_{1}$ and $\Gamma_{2}$. The exogenous concentration tapers off just in the vicinity where the endogenous concentration experiences a sudden increase over its background value of $g_{o}$. This local increase is caused by the compression produced by the rapidly advancing exogenous front. This compression decreases the local surface tension, giving rise to an additional Marangoni stress which opposes the direction of spreading. This effect reduces the spreading exponent and slows the advance of the surfactant film (Grotberg et al. 

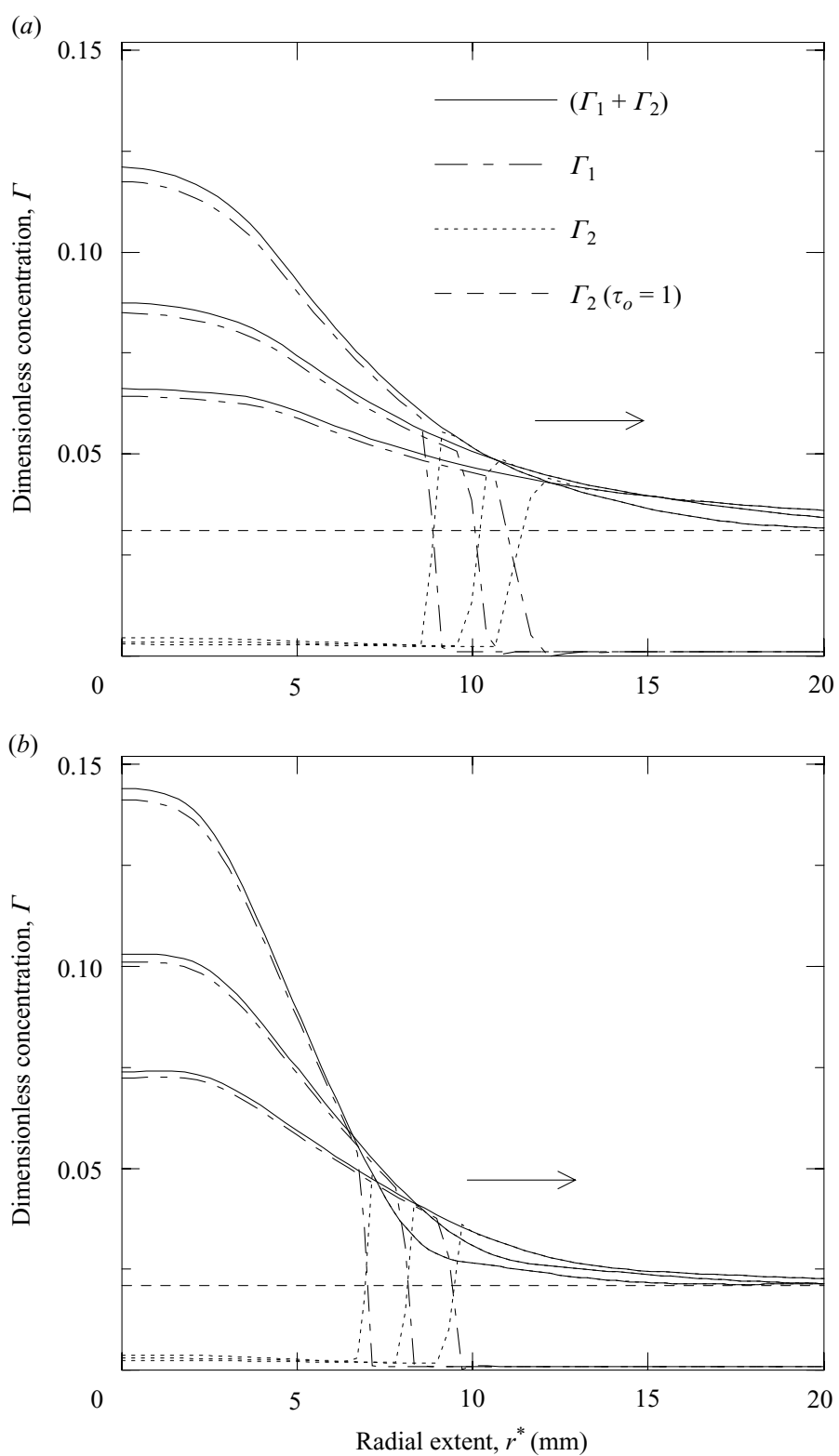

FIGURE 13. Numerical solutions for the total surfactant concentration, $\Gamma$, the exogenous concentration, $\Gamma_{1}$, and the endogenous concentration, $\Gamma_{2} . \tau_{o}=1$ represents origin of time. (a) Case 1B: $t^{*}=0.5,1.13$ and $2.5 \mathrm{~s}$. (b) Case 3C: $t^{*}=0.5,1.17$ and $3.0 \mathrm{~s}$. Parameter values for case $1 \mathrm{~B}$ and $3 \mathrm{C}$ given in table 2 . Arrows indicate direction of increasing time.

1995; Bull et al. 1999; Bull \& Grotberg 2003). By comparing the results of figure 13 with the those of figures 9 and 11, it is evident that the leading edge of the surfactant film, as defined by (4.13), is located behind the position of the minimum in the surface slope or, likewise, behind the location of the peak in the advancing rim. By examining the spreading relation found experimentally for the talc particles, they too locate behind the advancing rim, suggesting that the talc follows closely the behaviour of the advancing surfactant front. 


\begin{tabular}{lcccccc}
\hline & $K_{\min }$ & $a_{\min }$ & $r_{\min }$ & $K_{t}$ & $a_{t}$ & $r_{t}$ \\
Case 1A & 16.49 & 0.25 & 0.998 & 12.80 & 0.24 & 0.999 \\
Case 1B & 16.78 & 0.26 & 0.999 & 9.49 & 0.15 & 0.985 \\
Exp. 1 & $16.88 \pm 0.27$ & $0.25 \pm 0.02$ & $\ldots$ & $\mathrm{n} / \mathrm{a}$ & $\mathrm{n} / \mathrm{a}$ & $\mathrm{n} / \mathrm{a}$ \\
Case 3A & 14.73 & 0.26 & 0.999 & 8.08 & 0.15 & 0.990 \\
Case 3B & 15.33 & 0.26 & 0.999 & 8.67 & 0.16 & 0.990 \\
Case 3C & 13.60 & 0.26 & 0.999 & 7.63 & 0.18 & 0.991 \\
Exp. 3 & $13.61 \pm 0.16$ & $0.21 \pm 0.01$ & 0.989 & $8.81 \pm 0.23$ & $0.21 \pm 0.01$ & 0.997
\end{tabular}

TABLE 3. Comparison of theoretical and experimental fits to power-law expression for slope minimum (min) and talc front $(t)$. Variables defined in table 1.

\subsection{Prediction of spreading rates}

Values of $R_{\min }$ and $R_{t}$ were tabulated during each computational run and fitted to expressions of the form $R_{\min }=K_{\min } t^{a_{\min }}$ and $R_{t}=K_{t} t^{a_{t}}$; here the subscript $t$ signifies quantities associated with the talc front. The spreading coefficients and exponents were then obtained from a least-squares fit of numerical data spanning approximately three decades in time. The values obtained are shown in table 3. Case 1B agrees remarkably well with experimental values. Case $3 \mathrm{C}$ accurately predicts the coefficients, but the computed exponents lie below or above the experimental value. There are two differences between these experiments. The deposited volume of oleic acid was larger in run 3 and the liquid surface also contained talc particles. Since both runs contained an excess of oleic acid at the origin, the difference in deposited volume is not considered significant. There remains a question, however, as to whether the talc influences the flow by introducing inertial effects or spurious surface contamination. Our results suggest that the technique of locating the leading edge of an advancing monolayer by sprinkling talc particles on the surface of a shallow liquid layer affects the value of the measured spreading exponent. In the experiments with the talc, the spreading exponents were lower than the theoretical value of $1 / 4$. This may suggest that additional contamination by the talc produced a further surfactant compression effect ahead of the spreading oleic front, thereby slowing the overall spreading rate for $R_{\min }$ and $R_{t}$.

\section{Discussion and conclusion}

The surface deformation produced by the spreading of oleic acid on a thin aqueous mixture of glycerol has been investigated using area-wide Moiré topography, a simple and effective technique for measuring the slope of a liquid interface over macroscopic distances. The liquids used provided a model system for studying the spreading of an insoluble surfactant on a thin liquid film. The experimental profiles were integrated to reveal the surface deformation throughout the spreading process. These profiles reveal a minimum in the surface slope which corresponds to a point in the spreading film just ahead of an advancing rim. The deflection of the liquid interface decreases in time as the driving force for spreading diminishes. The experimental profiles can also be used to determine the rate of advance of the spreading monolayer. Both the coefficients and exponents for the spreading process can be compared with theoretical predictions. For the experiments described in this paper, we have shown that agreement between theory and experiment is quite good provided the model allows for a very small amount of pre-existing contaminant on the liquid surface. Despite careful and extensive cleaning 
procedures, the fact that the experiment was conducted in an area surrounded by optical and video equipment may have introduced some spurious contamination onto the glycerol-water surface. Nonetheless, inclusion of this feature into the model produced accurate predictions for the surface slope, surface deflection and spreading rates, especially for times $t^{*} \geqslant 1 \mathrm{~s}$. The agreement in the surface slope and shape at early times is also fairly good, though the model equations overestimate the value of slope maximum ahead of the deposited drop. This discrepancy suggests that the initial shear stress experienced by the liquid layer is smaller than predicted.

This overestimate may be caused by some differences between the theoretical assumptions of the model and the actual deposition procedure used in the experimental work. As a first consideration, the model equations describe the spreading of a surfactant monolayer along the surface of a thin liquid layer. Experimentally, however, the surfactant is delivered from a small droplet whose initial radius is larger than the film thickness on which it spreads. The lubrication approximation may therefore be violated during the very early stages of spreading. The last entry in table 2 gives the values of $\varepsilon R e$ corresponding to the parameter values used in computing the numerical profiles. The magnitude of the product $\varepsilon R e$ is based on the initial and largest values for the lubrication parameter and the Reynolds number. This product must be vanishingly small in order for inertial effects to be negligible. Although this will certainly be true at later times, the estimates shown indicate that inertial effects are order one at early times. Without performing the actual calculations, it is unclear at this time what would be the net effect of this contribution. As a second consideration, the capillary forces in the vicinity of the droplet edge, which are ignored in the theoretical model, can reduce the initial shear stress by pumping more liquid into the spreading film. This can also be accomplished by the presence of hydrostatic terms since the initial droplet height is comparable to the undisturbed film thickness. These effects would all soften the degree of film thinning observed, theoretically leading to a smaller value of the surface slope ahead of the deposited droplet. Another reason which could account for the smaller slope observed experimentally involves the assumption of insolubility. The solubility of an oleic acid monolayer subject to various degrees of compression for a pure water at $\mathrm{pH}$ values approximately less than 5.6 has been measured to be very small (Tomoaia-Cotisel et al. 1987). The addition of glycerol to an acidic subphase only slightly decreases the spreading pressure (Lim \& Berg 1975). It is possible that a smaller driving force for spreading would produce a smaller initial surface slope upstream. Finally, we note that in contrast to the experimental situation in which a macroscopic drop of oleic acid is deposited on the interface, the present model is based on the spreading of a dilute surfactant monolayer. Thus, in the real situation, the relatively large surfactant surface concentration present in the centre of the drop would cause the Marangoni stresses to diminish substantially in that region as predicted by a nonlinear equation of state. This physical scenario may reduce the spreading rate and therefore obviate the need to invoke the presence of endogenous surfactant.

Since many of the dimensionless numbers used to generate the numerical profiles depend on the lubrication parameter, $\varepsilon$, it is very important that this ratio be known quite accurately. For example, in our work we estimated the thickness of the undisturbed film from the volume of liquid poured into the sample dish. Because of the curvature at the bottom sides of the sample dish and wetting effects along the meniscus region, this estimate may have been in error by approximately $4 \%$. A more accurate technique must be developed to determine the exact thickness of the initial liquid layer, especially for very thin films. It would also be preferable to deliver the 


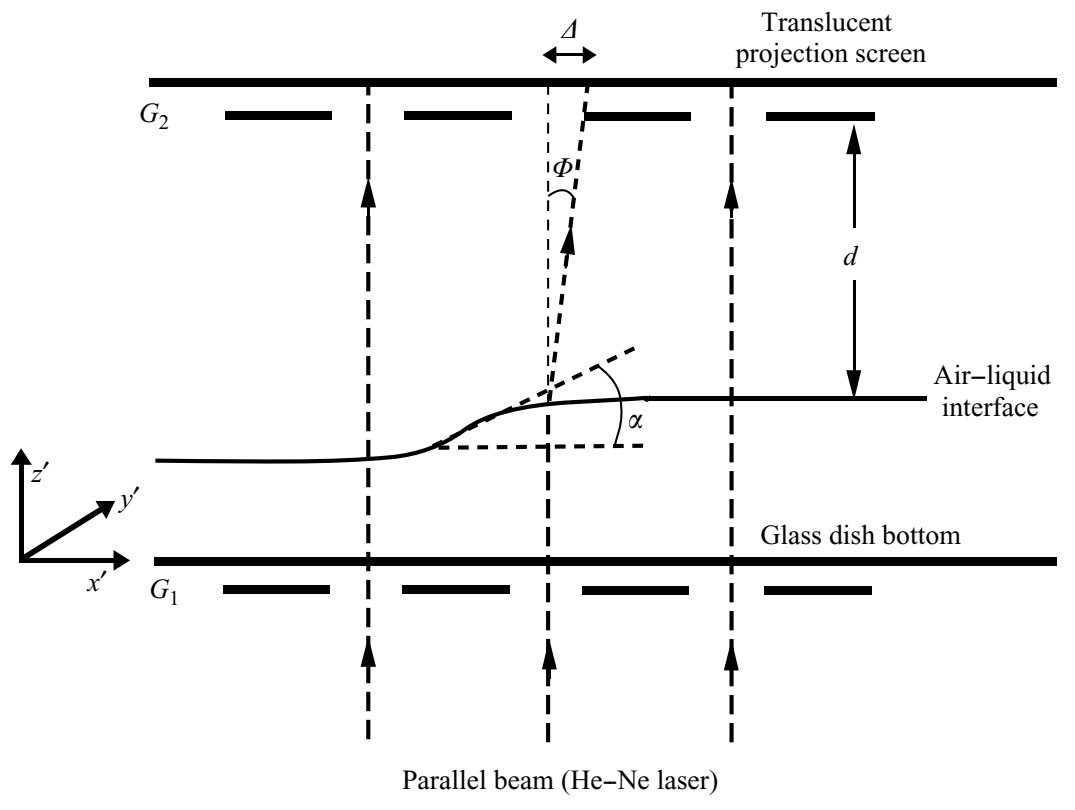

FIGURE 14. Deflection of light rays caused by surface distortion of initially flat liquid film. Local surface slope denoted by $\alpha$ where $\alpha \ll 1$.

surfactant as a true monolayer and not as a droplet. A Langmuir trough arrangement would allow a monolayer with a given spreading pressure and initial extent to be delivered exactly. Unfortunately, this technique is not viable for very thin films. In the absence of any such improvements to the experimental procedure, the comparison between theory and experiment could be made more direct by deriving a model which describes the deposition of an actual droplet of surfactant.

This work was supported by the National Science Foundation (Chemical and Transport Systems), the NASA Microgravity Fluid Physics Program and the Princeton Research Institute for the Science and Technology of Materials. S. M. T. also kindly acknowledges the Moore Distinguished Scholar Program at the California Institute of Technology.

\section{Appendix A}

Here, we provide details of the equations used to construct the time-varying flow profiles using the Moire technique. When the light traverses a uniformly flat liquid film, the projection of the illuminated grating, $G_{1}$, onto the reference grating, $G_{2}$, produces a series of alternating parallel black and white Moire fringes whose geometric origin is shown in figure 14. For dense line gratings and small opening angles $\theta$, the fringes appear as smooth continuous lines. As an example, figures $1(c)$ and $1(d)$ depict the fringes produced by our optical arrangement before and after droplet deposition. For the coordinate system shown in figure 14, the $y$-coordinates of the grating lines for $G_{1}$ and $G_{2}$ are described by

$$
\begin{aligned}
& y_{G_{1}}=-x \cot \frac{1}{2} \theta+\frac{1}{2} m p \operatorname{cosec} \frac{1}{2} \theta, \\
& y_{G_{2}}=-x \cot \frac{1}{2} \theta+\frac{1}{2} n p \operatorname{cosec} \frac{1}{2} \theta,
\end{aligned}
$$


where $m$ and $n$ represent the order of the grating line and assume integer values $\pm 1, \pm 2, \ldots, p$ is the pitch of the two identical gratings and $\theta$ the opening angle. The geometrical loci of intersection points between the two sets of gratings form an array of parallel bright fringes whose $y$-coordinate is defined by

$$
y=\frac{(m-n) p}{2 \sin \frac{1}{2} \theta} .
$$

The distance between successive fringes, $i$, is related to the pitch and the opening angle through the relation

$$
i=\frac{p}{2 \sin \frac{1}{2} \theta} .
$$

In our studies, typical values for these quantities were $p=0.025 \mathrm{~cm}, \theta=3.02^{\circ}$ and $i=0.475 \mathrm{~cm}$. As shown in figure 3 , when the liquid surface is deformed in the direction perpendicular to the rulings of the illuminated grating, $G_{1}$, the light is refracted away from the normal by a distance $\Delta=d \tan \phi$ where $d$ is the vertical distance between the liquid surface and the grating $G_{2}$, and $\phi$ represents the angular deflection from the normal. The position of the grating lines emanating from $G_{1}$, which have been refracted by the surface distortion, is then given by

$$
y=\frac{\cos \frac{1}{2} \theta}{\sin \frac{1}{2} \theta} x-\frac{n p}{\sin \frac{1}{2} \theta}-\frac{\Delta}{2 \sin \frac{1}{2} \theta} .
$$

The position of the resultant Moire fringes are therefore described by

$$
y=\frac{(m-n) p}{2 \sin \frac{1}{2} \theta}-\frac{\Delta}{2 \sin \frac{1}{2} \theta} .
$$

The last term defines the deviation of a fringe, $\delta y$, of order $m-n$ from its undisturbed position and can be re-expressed according to

$$
\delta y=\frac{\Delta}{2 \sin \frac{1}{2} \theta}=\frac{d \tan \phi}{2 \sin \frac{1}{2} \theta} .
$$

This equation illustrates that while the light deflection caused by the film surface distortion, $\Delta$, can be quite small, the deformation in the Moiré fringe can be quite large when the opening angle between the gratings is small. Using Snell's law, the deflection angle, $\phi$, can be directly related to the indices of refraction of the air and liquid substrate, as well as the surface slope, which is the quantity of interest. According to figure 3, for small surface slopes, $\alpha=\partial h / \partial x^{\prime}$,

$$
\phi=\sin ^{-1}\left(\frac{n_{\text {liquid }}}{n_{\text {air }}} \sin \alpha\right)-\alpha \simeq \alpha\left(\frac{n_{\text {liquid }}}{n_{\text {air }}}-1\right),
$$

where $n_{\text {liquid }}$ and $n_{\text {air }}$ are the refractive indices of the liquid substrate (glycerol-water mixture) and air. The surface slope of the distorted liquid film can be conveniently expressed in terms of measurable quantities. For small opening angles, $\theta$, the directions $\hat{x}$ and $\hat{x}^{\prime}$ in figure 14 are practically coincident. For small $\alpha$, the deflection angle, $\phi$, is also small. Using this small slope approximation and combining (4), (7) and (8) leads to:

$$
\frac{\partial h}{\partial x}=\frac{p \delta y}{i d\left(n_{\text {liquid }}-1\right)} .
$$

The distance $d$ is chosen so as to sample appropriate magnitudes in the surface slope. In our studies of a spreading monolayer, the choice $d \simeq 2 \mathrm{~cm}$ was sufficient to sample 
the majority of the surface slope during the spreading process (except the portion close to deposited droplet). For one set of experiments, we improved the resolution in measuring $\delta y$ by increasing $d$ to a distance of $7 \mathrm{~cm}$, as suggested by (A 7).

\section{Appendix B}

The relation describing the advance of a finite amount of insoluble surfactant spreading on a thin viscous layer in axisymmetric geometry is simply derived as follows. This derivation assumes there is no pre-existing surface-active contaminant (endogenous surfactant) on the initial liquid layer. Within the lubrication approximation, the tangential stress condition at the air-liquid interface dictates that the viscous stress be balanced by the gradient in surface tension according to

$$
\mu^{*} u_{z^{*}}^{*} \sim\left(\mu^{*} / H_{o}^{*}\right) d R^{*}\left(t^{*}\right) / d t^{*}=\sigma_{r^{*}}^{*},
$$

where $R^{*}\left(t^{*}\right)$ represents the extent of the monolayer at time $t^{*}$. For dilute surfactant concentrations, the equation of state relating the surface tension to the surface concentration can be written as $\sigma^{*}=\sigma_{o}^{*}-\beta \Gamma^{*}$ where $\beta=\partial \sigma^{*} / \partial \Gamma^{*}$ is a constant and $\beta \Gamma_{m}^{*}=\Pi^{*}$. The quantity $\Gamma_{m}^{*}$ denotes the uniform concentration of the deposited monolayer and $\Pi^{*}$ the spreading pressure. The gradient in surface tension is approximated by $\sigma_{r^{*}}^{*}=\sigma_{\Gamma^{*}}^{*} \Gamma_{r^{*}}^{*} \sim \beta\left(\Gamma^{*} / R^{*}\right)$. This relation can be recast in terms of the total mass of surfactant, $M^{*}$, defined by

$$
M^{*}=2 \pi \int_{0}^{\infty} r^{*} \Gamma^{*} \mathrm{~d} r^{*},
$$

from which the scaling $\Gamma^{*} \sim M^{*} / R^{* 2}$ is obtained. Equations (B1) and (B2) are combined to yield:

$$
\frac{\mu^{*}}{H_{o}^{*}} \frac{d R^{*}\left(t^{*}\right)}{d t^{*}} \sim \beta \frac{M^{*}}{R^{* 3}},
$$

which, when integrated, produces the relation:

$$
R^{*}\left(t^{*}\right)=c\left(\frac{4 \beta M^{*} H_{o}^{*}}{\mu^{*}}\right)^{1 / 4} t^{* 1 / 4},
$$

where $c$ is an undetermined constant. This relation was first derived by Jensen \& Grotberg (1992) and Espinosa et al. (1993).

Rewriting (B 2) in dimensionless form by introducing $r=r^{*} / R_{o}^{*}$ and $\Gamma=\Gamma^{*} / \Gamma_{m}^{*}$ gives:

$$
M^{*}=R_{o}^{* 2} \Gamma_{m}^{* 2} \pi \int_{0}^{\infty} r \Gamma \mathrm{d} r .
$$

For an initial surfactant distribution which resembles a top-hat function of unit amplitude that vanishes at $r=1$, the integral is equal to $1 / 2$ and (B 5) becomes

$$
M^{*}=\pi R_{o}^{* 2} \Gamma_{m}^{*} .
$$

Substituting $\beta M^{*}=\beta \Gamma_{m}^{*} \pi R_{o}^{* 2}=\Pi^{*} \pi R_{o}^{* 2}$ into (B 4) yields the desired equation:

$$
R^{*}(t)=c\left(\frac{4 \pi R_{o}^{* 2} \Pi^{*} H_{o}^{*}}{\mu^{*}}\right)^{1 / 4} t^{* 1 / 4} .
$$

This relation implies that a decrease in $\mu^{*}$ or an increase in $H_{o}^{*}, \Pi^{*}$ or $R_{o}^{*}$, produces more rapid spreading of the surfactant monolayer. The $1 / 4$ exponent remains valid 
provided that the tangential stress at the air-liquid interface caused by the gradient in surface tension is mainly responsible for the spontaneous spreading. The inclusion of significant capillary and hydrostatic terms will modify the spreading exponent, as can the presence of endogenous surface-active species.

\section{REFERENCES}

Adamson, A. W. 1990 Physical Chemistry of Surfaces. J. Wiley, New York.

Afsar-Siddiqui, A. B., Luckham, P. F. \& Matar, O. K. $2003 a$ Unstable spreading of aqueous anionic surfactant solutions on liquid films. Part 1. Sparingly soluble surfactant. Langmuir 19, 696-702.

Afsar-Siddiqui, A. B., Luckham, P. F. \& Matar, O. K. $2003 b$ Unstable spreading of aqueous anionic surfactant solutions on liquid films. Part 1. Sparingly soluble surfactant. Langmuir 19, 703-708.

Ahmad, J. \& HANSEN, R. S. 1972 A simple quantitative treatment of the spreading of monolayers on thin liquid films. J. Colloid Interface Sci. 38, 601.

Bull, J. L. \& Grotberg, J. B. 2003 Surfactant spreading on thin viscous films: film thickness evolution and periodic wall stretch. Exps. Fluids 34, 1.

Bull, J. L., Nelson, L. K., Walsh, J. T., Glucksberg, M. R., Schurch, S. \& Grotberg, J. B. 1999 Surfactant-spreading and surface-compression disturbance on a thin viscous film. J. Biomech. Engng 121, 88.

CAChile, M. \& CAZABat, A. M. 1999 Spontaneous spreading of surfactant solutions on hydrophilic surfaces: CnEn in ethylene and diethylene glycol. Langmuir 15, 1515.

Cachile, M., Cazabat, A. M., Bardon, S., Valignat, M. P. \& Vadenbrouck, P. 1999 Spontaneous spreading of surfactant solutions on hydrophilic surfaces. Colloids Surf. A 159, 47.

Cachile, M., Schneemlich, M., Hamraoui, A. \& Cazabat, A. M. 2002 Films driven by surface tension gradients. Adv. Colloids Interface Sci. 96, 59.

CAmP, D. W. \& BerG, J. 1987 The spreading of oil on water in the surface tension regime. J. Fluid Mech. 184, 445.

DaGan, Z. 1984 Spreading of films of adsorption on a liquid surface. Physico Chem. Hydrodyn. 5, 43.

DiPietro, N. D., Huh, C. \& Cox, R. 1978 The hydrodynamics of the spreading of one liquid on the surface of another. J. Fluid Mech. 84, 529.

Dussaud, A. D. \& Troian, S. M. 1997 Dynamics of spontaneous spreading with evaporation on a deep fluid layer. Phys. Fluids 10, 23.

Espinosa, F. F., Shapiro, A. H., Fredberg, J. J. \& Kamm, R. D. 1993 Spreading of exogenous surfactant in an airway. J. Appl. Physiol. 75, 2028.

FAY, J. A. 1969 The spread of oil slicks on a calm sea. In Oil on the Sea (ed. D. Hoult). Plenum Press, New York.

Fisher, B. J., Darhuber, A. A. \& Troian, S. M. 2001 Streamlets and branching dynamics in surfactant driven flows. Phys. Fluids 13, 9.

FoDA, M. \& Cox, R. 1980 The spreading of thin liquid films on a water-air interface. J. Fluid Mech. 101, 33.

Fraaije, J. G. E. M. \& Cazabat, A. M. 1989 Dynamics of spreading on a liquid substrate. J. Colloid Interface Sci. 133, 452.

Gaver, D. P. \& Grotberg, J. B. 1990 The dynamics of a localized surfactant on a thin film. J. Fluid Mech. 213, 127.

Gaver, D. P. \& Grotberg, J. B. 1992 Droplet spreading on a thin viscous film. J. Fluid Mech. 235, 399.

Grotberg, J. B., Halpern, D. \& Jensen, O. E. 1995 Interaction of exogenous and endogenous surfactant: spreading-rate effects. J. Appl. Physiol. 78, 750.

HARPER, J. F. 1992 The leading edge of an oil slick, soap film, or bubble stagnant cap in stokes flow. J. Fluid Mech. 237, 23.

Harper, J. F. \& Dixon, J. N. 1974 The leading edge of a surface film on contaminated water. Proc. Fifth Australasian Conf. Hydraul. Fluid Mech. 2, 499.

Hoult, D. 1972 Oil spreading on the sea. Annu. Rev. Fluid Mech. 4, 341. 
Huh, C., Inoue, M. \& Mason, S. 1975 Unidirectional spreading of one liquid on the surface of another. Can. J. Chem. Engng 53, 367.

Hussain, Z., Fatima, M. \& Ahmad, J. 1975 The rate of spreading of monolayers on liquids. J. Colloid Interface Sci. 50, 44.

JENSEN, O. E. 1995 The spreading of insoluble surfactant at the free surface of a deep fluid layer. J. Fluid Mech. 293, 349.

Jensen, O. E. \& Grotberg, J. B. 1992 Insoluble surfactant spreading on a thin viscous film: shock evolution and rupture. J. Fluid Mech. 240, 259.

Jungermann, E. \& Sonntag, N. O. V. 1991 Glycerine. Marcel Dekker.

Kafri, O. \& Glatt, I. 1989 The Physics of Moiré Metrology. John Wiley, New York.

Kheshgi, H. S. \& Scriven, L. E. 1983 Measurement of liquid film profiles by Moiré topography. Chem. Engng Sci. 38, 525.

LiM, Y. C. \& BerG, J. C. 1975 Surface presssure - area behavior of fatty acid monolayers on multicomponent substrates. J. Colloid Interface Sci. 51, 162.

Marmur, A. \& Lelah, M. D. 1981 The spreading of aqueous surfactant solutions on glass. Chem. Engng Commun. 13, 133.

Nikolov, A. D., Wasan, D. T., Chengara, A., Koczo, K., Policello, G. A. \& Kollosvary, I. 2002 Superspreading driven by marangoni flow. Adv. Colloid Interface Sci. 96, 59.

Schiesser, W. E. 1991 The Numerical Method of Lines. Academic Press, San Diego.

Sсотт, J. C. 1972 The influence of surface-active contamination on the initiation of wind waves. J. Fluid Mech. 56, 591.

Scott, J. C. \& Stephens, R. W. B. 1972 Use of moiré fringes in investigating surface wave propagation in monolayers of soluble polymers. J. Acoust. Soc. Am. 52, 871.

Stoebe, T., Lin, Z., Hill, R. M., Ward, M. D. \& Davis, H. T. 1997 a Enhanced spreading of aqueous films containing ethoxylated alcohol surfactants on solid substrates. Langmuir 13, 7270.

Stoebe, T., Lin, Z., Hill, R. M., Ward, M. D. \& Davis, H. T. $1997 b$ Enhanced spreading of aqueous films containing ionic surfactants on solid substrates. Langmuir 13, 7276.

Theocaris, P. S. \& Koutsabessis, A. 1965 Shape measurement by means of Moiré fringes. J. Sci. Instrum. 42, 607.

Tomoaia-Cotisel, M., Zsako, J., Mocanu, A., Lupea, M. \& Chifu, E. 1987 Insoluble mixed monolayers. 3. The ionization characteristics of some fatty-acids at the air-water interface. J. Colloid Interface Sci. 117, 464.

Troian, S. M., Wu, X. L. \& Safran, S. A. 1989 Fingering instability in thin wetting films. Phys. Rev. Lett. 62, 1496. 